\title{
Projective-injective modules, Serre functors and symmetric algebras
}

\author{
Volodymyr Mazorchuk and Catharina Stroppel
}

\begin{abstract}
We describe Serre functors for (generalisations of) the category $\mathcal{O}$ associated with a semi-simple complex Lie algebra. In our approach, projective-injective modules, that is modules which are both, projective and injective, play an important role. They control the Serre functor in the case of a quasi-hereditary algebra having a double centraliser given by a projective-injective module whose endomorphism ring is a symmetric algebra. As an application of the double centraliser property together with our description of Serre functors, we prove three conjectures of Khovanov about the projective-injective modules in the parabolic category $\mathcal{O}_{0}^{\mu}\left(\mathfrak{s l}_{n}\right)$.
\end{abstract}

\section{Contents}

1 Introduction $\quad 2$

2 Standardly stratified structure and double centralisers $\quad 6$

2.1 Tilting modules and Ringel duality . . . . . . . . . . . 7

2.2 Double centraliser property . . . . . . . . . . . . 9

2.3 Double centralizer and projective-injective modules . . . . . . 10

3 Serre functors $\quad 13$

3.1 A characterisation of Serre functors . . . . . . . . . . . . . 14

3.2 Serre functors via partial coapproximation . . . . . . . . . . 16

4 Applications $\quad 19$

4.1 Bernstein-Gelfand-Gelfand category $\mathcal{O} \ldots \ldots$. . . . . . . . 19

4.2 The parabolic category $\mathcal{O}$ in the sense of Rocha-Caridi . . . . 22

4.3 Harish-Chandra bimodules . . . . . . . . . . . . . . . . . 29

4.4 The category $\mathcal{O}$ for rational Cherednik algebras . . . . . . . 30

$5 \quad$ Projective-injectives in the category $\mathcal{O}^{\mathfrak{p}}\left(\mathfrak{s l}_{n}(\mathbb{C})\right) \quad 32$

5.1 On a result of Irving . . . . . . . . . . . . . . . . . . 33

5.2 On Khovanov's conjectures . . . . . . . . . . . . . . 34 


\section{Introduction}

Symmetric algebras are particularly well-behaved algebras with several special properties. We first want to recall two of these properties and then discuss to which extend they can be generalised and transferred to other finite dimensional algebras. A finite dimensional algebra $A$ is called symmetric if there is an $A$-bimodule isomorphism $A \cong A^{*}$. In particular, projective $A$-modules are always injective. On the other hand, since $A \cong A^{*}$ as $A$-bimodules, the isomorphism of vector spaces

$$
\operatorname{Hom}_{A}(A, A) \cong \operatorname{Hom}_{A}\left(A, A^{*} \otimes_{A} A\right) \cong \operatorname{Hom}_{A}(A, A)^{*}
$$

gives rise to an isomorphism

$$
\operatorname{Hom}_{A}(M, N) \cong \operatorname{Hom}_{A}\left(M, A^{*} \otimes_{A} N\right) \cong \operatorname{Hom}_{A}(N, M)^{*}
$$

for any free $A$-modules $M$ and $N$. Moreover, this isomorphism is functorial in $M$ and $N$.

The question we want to ask now is whether these two properties can be transferred somehow to a more general class of algebras. We will consider finite dimensional algebras which have a symmetric subalgebra which, in some sense, can control the representation theory of the original algebra. If we are lucky, $A$ has "enough" projective modules which are also injective. We fix a system of representatives for the isomorphism classes of indecomposable projective-injective (i.e. at the same time projective and injective) $A$-modules. Then, instead of considering $A$ itself we propose to consider the direct sum, say $Q$, of all modules from this fixed system. The endomorphism ring $\operatorname{End}_{A}(Q)$ is at least a Frobenius algebra. The following questions naturally arise

- Is $\operatorname{End}_{A}(Q)$ a symmetric algebra?

- How much information about the category of finitely generated $A$ modules is already encoded in $Q$ and $\operatorname{End}_{A}(Q)$ ?

- Is there an isomorphism, functorial in both entries,

$$
\operatorname{Hom}_{A}\left(P_{1}, P_{2}\right) \cong \operatorname{Hom}_{A}\left(P_{1}, A^{*} \otimes_{A} P_{2}\right) \cong \operatorname{Hom}_{A}\left(P_{2}, P_{1}\right)^{*}
$$

for any projective-injective $A$-modules $P_{1}$ and $P_{2}$ ?

In general, the first question is very difficult to answer. Concerning the second question, we will describe the situation where all the information about $A$-mod is already contained in $Q$ and $\operatorname{End}_{A}(Q)$. This is given by the so-called double centraliser property which we will explain more precisely 
shortly. The last question relates to the existence and description of a socalled Serre functor, motivated by Serre's duality for sheaves on projective varieties. The purpose of this paper is to answer the above three questions for certain algebras appearing in representation theory.

To substantiate and to specify our approach we would like to recall the important role projective-injective modules play in representation theory, in particular in different versions of the category $\mathcal{O}$. The striking example is the case of an integral block of the category $\mathcal{O}$ for a semisimple complex Lie algebra. By Soergel's result ([Soe90, Endomorphismensatz and Struktursatz]) such a block can be completely described by (a certain subcategory of) the category of finitely generated modules over the endomorphism ring of the (unique up to isomorphism) indecomposable projective-injective module in this block. This idea was generalised and formalised in [KSX01] as the socalled double centraliser property. In this language, Soergel's result could be stated as follows: Let $A$ be a finite dimensional algebra such that $A$-mod is equivalent to the block of the category $\mathcal{O}$ in question. Then for the indecomposable projective-injective $A$-module $Q$ we have $A=\operatorname{End}_{\operatorname{End}_{A}(Q)}(Q)$. Note that [Soe90, Endomorphismensatz] implies that $\operatorname{End}_{A}(Q)$ is a symmetric algebra.

Using the Ringel duality functor it is easy to see (Corollary 2.4) that there is always a tilting module $T$ having the double centraliser property above. This module $T$ need not to be projective-injective in general. Nevertheless, there are many known examples where we have the particularly handy situation of the double centraliser property with respect to a projectiveinjective tilting module $T$. We recall such examples in Section 2. Since in these cases the category $A$-mod is completely determined by $\operatorname{End}_{A}(T)$ and $T$, it follows directly that the centre of $A$-mod is isomorphic to the centre of the endomorphism ring of $T$ (in particular [Kho04, Conjecture 4] follows, see Theorem 5.2).

Motivated by Serre's duality, there is the notion of a Serre functor for any $\mathbb{k}$-linear category with finite dimensional homomorphism spaces (see Section 3). Kapranov and Bondal ([BK89]) showed that the bounded derived category $\mathcal{D}^{b}(A)$ for any finite dimensional algebra $A$ of finite global dimension admits a Serre functor. In fact, the existence of a Serre functor is equivalent to the finiteness of the global dimension of $A$ and also to the existence of Auslander-Reiten triangles ([RVdB02], [Hap91]). It is well-known that in the latter case the Serre functor is the left derived of the Nakayama functor (see e.g. [Hap88, page 37]), that is of the functor isomorphic to $A^{*} \otimes_{A} \bullet$. However, if the algebra $A$ is not explicitly given, the Serre functor might be hard to compute.

Nevertheless, in some cases the Serre functor for $\mathcal{D}^{b}(A)$ can be explicitly described, using for instance geometric or functorial methods. For example, 
in [BK89] it was conjectured that the Serre functor of the bounded derived category of perverse sheaves on flag varieties is given by a geometrically defined intertwining functor. This was our motivation to study the Serre functor of the bounded derived category of (integral blocks of) $\mathcal{O}$, associated with the corresponding semi-simple Lie algebra, which is equivalent to the category of perverse sheaves in question. The original conjecture has recently been proved by Beilinson, Bezrukavnikov and Mirkovic in [BBM04]. In the present paper we explicitly construct the Serre functor for the bounded derived category of any integral block of $\mathcal{O}$ using the twisting functors, defined in [Ark97] and studied e.g. in [AS03]. Our approach is purely algebraic and does not require the explicit knowledge of the associative algebra associated to $\mathcal{O}$. As a (very unexpected) consequence we obtain an isomorphism between a certain composition of twisting functors and a certain composition of Irving's shuffling functors (see Corollary 4.2).

We further apply this result to construct the Serre functors of the bounded derived categories of (integral blocks of) of any parabolic category $\mathcal{O}^{\mathfrak{p}}$ in the sense of Rocha-Caridi, [RC80]. Using the explicit description of the Serre functor (in terms of shuffling functors) we prove in Theorem 4.6 that the endomorphism algebra of the sum of all indecomposable projective-injective modules in $\mathcal{O}^{\mathfrak{p}}$ is symmetric, which was conjectured by Khovanov. One of the motivations to consider the category of projective-injective modules in $\mathcal{O}^{\mathfrak{p}}$ is to find a precise connection between Khovanov's categorification of the Jones polynomial ([Kho00]) and the categorification of the Jones polynomial via representation theory of the Lie algebra $\mathfrak{s l}_{n}$ (as proposed in [BFK99] and proved in [Str05]). It might be possible to simplify the approach in [BFK99] and [Str05] by working with these symmetric endomorphism algebras. Moreover, from a topological point of view it seems to be much more natural and plausible to work with symmetric (or at least Frobenius) algebras to construct knot invariants instead of the complicated algebras which describe the integral blocks of the (parabolic) category $\mathcal{O}$.

We expect that the Serre functor for the category $\mathcal{O}$ for rational Cherednik algebras can be constructed in a similar way via twisting functors as the Serre functor for the Bernstein-Gelfand-Gelfand category $\mathcal{O}$. However, we are not able to prove this, mainly because of the lack of translation functors. Nevertheless, we give a description of the Serre functor for the category $\mathcal{O}$ for rational Cherednik algebras via partial coapproximation with respect to the direct sum of all indecomposable projective-injective modules (see $[\mathrm{KM} 05,2.5])$. The proof however uses the fact that Hecke algebras are symmetric and the properties of the Knizhnik-Zamolodchikov-functor. Using [GGOR03, Remark 5.10] it might be possible to construct the Serre functor in a different way, which would imply a conceptual proof of the fact ([CIK71]) that the Hecke algebras occurring here are symmetric (see Conjecture 4.12). 
In the last section of the paper we consider the special case of a parabolic category, $\mathcal{O}_{0}^{\mathfrak{p}}\left(\mathfrak{s l}_{n}\right)$, for the Lie algebra $\mathfrak{s l}_{n}$. For this category we give an easier proof of the main result of [Irv85] (Theorem 5.1). As a consequence we show that there is a double centraliser property with respect to a basic projective-injective module. This implies [Kho04, Conjecture 4]. The parabolic subalgebra $\mathfrak{p}$ of $\mathfrak{s l}_{n}$ is determined by some composition of $n$. In [Irv85, Proposition 4.3] it is shown that indecomposable projective-injective modules in $\mathcal{O}_{0}\left(\mathfrak{s l}_{n}\right)^{\mathfrak{p}}$ are indexed by the elements of some left cell. The Kazhdan-Lusztig combinatorics of translation functors, applied to these projective-injective modules, suggests a connection with Specht modules for the symmetric group $S_{n}$. It is well known that the Specht modules which correspond to different compositions of $n$, but giving rise to the same partition of $n$, are isomorphic. This observation might have led M. Khovanov to the conjecture that the endomorphism algebras of the basic projectiveinjective modules in different $\mathcal{O}_{0}\left(\mathfrak{s l}_{n}\right)^{\mathfrak{p}}$, corresponding to the same partition of $n$, are isomorphic. We finish the paper by proving this conjecture (Theorem 5.4).

Some guidance for the reader: Section 2 recalls some facts and results on double centralisers for module categories over standardly stratified algebras. In principle, the content is not new, the viewpoint might be slightly more general than usual. We formulated the setup as generally as possible, since we believe that our approach can be applied to a much wider class of algebras than the ones actually appearing in the paper. For the reader, however, it might be more approachable to have first a look at the Examples 2.7, skip the details of Section 2 and focus on the main result characterising Serre functors which can be found in Section 3. Applications and concrete descriptions of Serre functors are given in Section 4. The deepest result might be Theorem 4.6 stating that the endomorphism ring of a basic projective-injective module in the principal block of a (parabolic) category $\mathcal{O}$ is not only Frobenius, but symmetric.

Acknowledgements. We thank Mikhail Khovanov for sharing his ideas with us and for many helpful discussions and remarks. We also would like to thank Iain Gordon for many useful discussions and explanations and Ken Brown for useful remarks. We also thank Joshua Sussan for comments on a preliminary version of the paper. The first author was partially supported by The Royal Swedish Academy of Sciences, The Swedish Research Council, and STINT. The second author was supported by EPSRC. 


\section{Standardly stratified structure and double cen- tralisers}

In representation theory double centraliser properties play an important role. The aim of this section is to recollect known results from the literature, to emphasise the universal principle behind it, and to show the significance of projective-injective modules.

Let $\mathbb{k}$ be an algebraically closed field. Let $A$ be a unital finite dimensional associative $\mathbb{k}$-algebra. We denote by $A$-mod $(\bmod -A)$ the category of finite dimensional left (resp. right) $A$-modules. In the following we will mainly work with left $A$-modules, hence an " $A$-module" is always meant to be a left $A$-module. For $M, N \in A$-mod we denote by $\operatorname{Tr}_{M} N$ the trace of $M$ in $N$ (which is by definition the submodule of $N$, generated by the images of all morphisms from $M$ to $N)$.

Let $\{L(\lambda)\}_{\lambda \in \Lambda}$ be a complete set of representatives for the isomorphism classes of simple $A$-modules. For a simple $A$-module, $L(\lambda)$, we denote by $P(\lambda)$ its projective cover, and by $I(\lambda)$ its injective hull. We assume that there is a partial pre-order $\preceq$ (i.e. a reflexive and transitive binary relation) on $\Lambda$, which we fix. Let $P^{\succ \lambda}=\bigoplus_{\mu \succ \lambda} P(\mu)$ and $P^{\succeq \lambda}=\bigoplus_{\mu \succeq \lambda} P(\mu)$.

With respect to $\preceq$ we define the so-called standard module $\Delta(\lambda)$ to be the largest quotient of $P(\lambda)$ containing only composition factors of the form $L(\mu)$, where $\lambda \nprec \mu$, i.e. $\Delta(\lambda)=P(\lambda) / \operatorname{Tr}_{P \succ \lambda} P(\lambda)$. We also have a proper standard module $\bar{\Delta}(\lambda)$ which is the largest quotient of $P(\lambda)$ such that its radical contains only composition factors of the form $L(\mu)$, where $\lambda \npreceq \mu$, i.e. $\bar{\Delta}(\lambda)=P(\lambda) / \operatorname{Tr}_{P \succeq \lambda} \operatorname{rad} P(\lambda)$. Dually, we have the costandard module $\nabla(\lambda)$ and the proper costandard module $\bar{\nabla}(\lambda)$.

We denote by $\mathcal{F}\left(\Delta^{A}\right)=\mathcal{F}(\Delta)$ the full subcategory of $A$-mod given by all modules having a filtration, with all subquotients of this filtration being isomorphic to $\Delta(\lambda)$ for various $\lambda \in \Lambda$. If $M \in \mathcal{F}(\Delta)$ then we say that $M$ has a standard flag. Similarly, we define $\mathcal{F}(\bar{\Delta}), \mathcal{F}(\nabla), \mathcal{F}(\bar{\nabla})$, the categories of modules having a proper standard, a costandard, and a proper costandard flag respectively.

Let $A$ be a finite dimensional standardly stratified algebra as defined in [CPS96], that is

- the kernel of the canonical surjection $P(\lambda) \rightarrow \Delta(\lambda)$ has a standard flag;

- the kernel of the canonical surjection $\Delta(\lambda) \rightarrow L(\lambda)$ has a filtration with subquotients $L(\mu)$, where $\mu \preceq \lambda$.

In particular, if $\preceq$ is a partial order and $\Delta(\lambda)=\bar{\Delta}(\lambda)$ for $\lambda$, then $A$ is quasi-hereditary (see [CPS88, DR89]). If $\preceq$ is a partial order and any $\Delta(\lambda)$ has a proper standard flag, then $A$ is properly stratified (see [Dla00]). 
We call a module $M$ basic with respect to some property $\mathcal{P}$, if $M$ is the direct sum of pairwise non-isomorphic indecomposable modules with property $\mathcal{P}$ and any indecomposable module having this property is isomorphic to a summand in $M$. For example, a basic projective module in $A$-mod is a minimal projective generator. If $N=\oplus_{i=1}^{k} N_{i}^{m_{i}}$, where $m_{i} \in\{1,2, \ldots\}$ for all $i$, with $N_{i}$ indecomposable and pairwise non-isomorphic, we set $N_{\text {basic }}=$ $\oplus_{i=1}^{k} N_{i}$.

\subsection{Tilting modules and Ringel duality}

A tilting module is an object in $\mathcal{F}(\Delta) \cap \mathcal{F}(\bar{\nabla})$, and a cotilting module is an object in $\mathcal{F}(\bar{\Delta}) \cap \mathcal{F}(\nabla)$. In [Fri04] it is shown that for a standardly stratified algebra the category $\mathcal{F}(\Delta) \cap \mathcal{F}(\bar{\nabla})$ is closed under taking direct summands and that the indecomposable modules in this category are in natural bijection with standard modules. Let $T(\lambda)$ denote the unique indecomposable tilting module having a standard flag, where $\Delta(\lambda)$ occurs as a submodule. Let $T=\oplus_{\lambda \in \Lambda} T(\lambda)$ be the characteristic tilting module. There is the dual notion of cotilting modules. In general, cotilting modules cannot be classified in the same way as tilting modules. However, this can be done in the case when the opposite algebra $A^{o p p}$ is also standardly stratified (with respect to the same partial pre-order), see [Fri04, 4.2]. For quasi-hereditary algebras cotilting and tilting modules obviously coincide, but in general they do not have to. The Ringel duality functor (as introduced in [Rin91]) was studied in the more general setup of various stratified algebras for example in [ÁHLU00] and [Fri04]. We will need the following slight variation of these results:

Proposition 2.1. Let $A$ be a standardly stratified algebra. Then the Ringel dual $R(A)=\operatorname{End}_{A}(T)$ is standardly stratified and the contravariant functor $\mathrm{R}=\operatorname{Hom}_{A}(\bullet, T): A$-mod $\rightarrow \bmod -R(A)$ satisfies the following properties:

(1) $\mathrm{R}$ maps tilting modules to projective modules.

(2) $\mathrm{R}$ maps projective modules to tilting modules.

(3) $\mathrm{R}$ defines an equivalence of categories $\mathcal{F}\left(\Delta^{A}\right) \cong \mathcal{F}\left(\Delta^{R(A)}\right)$.

Proof. That the algebra $R(A)$ is standardly stratified follows for example from [Fri04, Theorem 5 (iii)]. Obviously, $T$ is mapped to $\operatorname{End}_{A}(T)$, hence it is projective. Taking direct summands implies the first statement. The last statement is proved analogously to [ÁHLU00, Theorem 2.6 (iv)] (note that the duality $\mathrm{D}$ used there swaps standard and costandard modules). To prove the second statement let now $Q$ be projective, then $\mathrm{R} Q$ has a standard flag. Of course, $\operatorname{Ext}_{A}^{1}(Q, \Delta(\lambda))=0$ for any $\lambda$. Using the last part of the proposition we get $\operatorname{Ext}_{R(A)}^{1}(\mathrm{R} \Delta(\lambda), \mathrm{R} Q)=0$, even $\operatorname{Ext}_{R(A)}^{1}\left(\Delta^{R(A)}(\lambda), \mathrm{R} Q\right)=0$ for 
any standard module $\Delta^{R(A)}(\lambda) \in R(A)$-mod. Therefore, (see e.g. [Fri04, Theorem 3] and [ÁHLU00, Theorem 1.6]), RQ has a proper costandard flag, hence it is tilting.

For any abelian category $\mathcal{C}$ we denote by $\mathcal{D}^{b}(\mathcal{C})$ its bounded derived category. If $\mathcal{C}=A$-mod we set $\mathcal{D}^{b}(A)=\mathcal{D}^{b}(\mathcal{C})$. If the opposite is not explicitly stated, by a "functor" we always mean a covariant functor. We use the standard notation like $\mathcal{L} F, \mathcal{R} G, \mathcal{L}_{i} F, \mathcal{R}^{i} G$ etc. to denote left derived and right derived functors and their $i$-th cohomology functors.

For the sake of completeness we mention the following fact

Proposition 2.2. Let $A$ and $B$ be standardly stratified such that tilting modules are also cotilting. Let $F: A-\bmod \rightarrow B-\bmod$ be a (covariant) rightexact functor with right adjoint $G$. Assume that $F$ defines an equivalence

$$
\mathcal{F}\left(\bar{\Delta}^{A}\right) \cong \mathcal{F}\left(\bar{\nabla}^{B}\right) .
$$

Then the following hold

(1) F maps projective modules to tilting modules and tilting modules to injective modules. In fact, $F$ defines equivalences (with inverse $G$ ) of the corresponding additive subcategories.

(2) If $A$ has finite global dimension then $B$ has finite global dimension as well, moreover, $\mathcal{L} F: \mathcal{D}^{b}(A) \rightarrow \mathcal{D}^{b}(B)$ is an equivalence with inverse $\mathcal{R} G$.

(3) $B$ is the Ringel dual of $A$.

Proof. Let $P \in A$-mod be projective, then $F P \in \mathcal{F}\left(\bar{\nabla}^{B}\right)$ by assumption and

$$
\operatorname{Ext}_{B}^{1}(F P, \bar{\nabla}(\lambda)) \cong \operatorname{Ext}_{B}^{1}\left(F P, F F^{-1} \bar{\nabla}(\lambda)\right) \cong \operatorname{Ext}_{A}^{1}\left(P, F^{-1} \bar{\nabla}(\lambda)\right)=0
$$

for any proper costandard module $\bar{\nabla}(\lambda)$. Hence $F P \in \mathcal{F}\left(\Delta^{B}\right)$ and is therefore tilting. If $X$ is tilting, hence cotilting, then $X \in \mathcal{F}\left(\bar{\Delta}^{A}\right)$. Therefore $F X \in \mathcal{F}\left(\bar{\nabla}^{B}\right)$ and

$$
\begin{aligned}
\operatorname{Ext}_{B}^{1}\left(\bar{\nabla}^{B}(\lambda), F X\right) & \cong \operatorname{Ext}_{B}^{1}\left(F F^{-1} \bar{\nabla}^{B}(\lambda), F X\right) \\
& \cong \operatorname{Ext}_{A}^{1}\left(F^{-1} \bar{\nabla}^{B}(\lambda), X\right)=0
\end{aligned}
$$

for any proper costandard module $\bar{\nabla}^{B}(\lambda)$, since $F^{-1} \bar{\nabla}^{B}(\lambda) \in \mathcal{F}\left(\bar{\Delta}^{A}\right)$ and $X \in \mathcal{F}\left(\nabla^{A}\right)$. If we now choose an inclusion of $F X \in \mathcal{F}\left(\bar{\nabla}^{B}\right)$ in its injective hull, then the cokernel is contained in $\mathcal{F}\left(\bar{\nabla}^{B}\right)$ and the inclusion splits because of (2.1). This means that $F X$ is injective and the first part follows. We have 
$\mathcal{R} G \mathcal{L} F \cong$ ID on projectives and $\mathcal{L} F \mathcal{R} G \cong$ ID on injectives. This implies that the global dimension of $B$ is finite and then the second statement follows. The fact that $B$ is the Ringel dual of $A$ is then clear from the definitions.

\subsection{Double centraliser property}

We claim that, given a standardly stratified algebra $A$, there is always some tilting module $X$ such that we have a double centraliser property, $A \cong$ $\operatorname{End}_{\operatorname{End}_{A}(X)}(X)$. This relies on the following

Proposition 2.3. Let $A$ be standardly stratified and let $R=R(A)$ be its Ringel dual. Let $P$ be the projective cover of the characteristic tilting module $T$ in mod- $R$. Then there is an exact sequence $0 \rightarrow A \rightarrow Q \rightarrow$ coker $\rightarrow 0$, where $Q=\mathrm{R}^{-1} P$ (see Proposition 2.1) is tilting and coker $\in \mathcal{F}\left(\Delta^{A}\right)$.

Proof. Since $P, T \in \mathcal{F}\left(\Delta^{R}\right)$, the kernel $K$ of the surjection between $P$ and $T$ is contained in $\mathcal{F}\left(\Delta^{R}\right)$ ([ÁHLU00, Theorem 1.6 (i)] and [Fri04, Theorem 3]). Applying the inverse of the Ringel duality functor (which is defined on $\mathcal{F}\left(\Delta^{R}\right)$ ) we get the short exact sequence

$$
0 \rightarrow A \rightarrow Q \rightarrow \text { coker } \rightarrow 0
$$

where coker $\in \mathcal{F}(\Delta)$ by Proposition 2.1.

Corollary 2.4. There exists a (basic) tilting module $X$ such that we have an isomorphism, $A \cong \operatorname{End}_{\operatorname{End}_{A}(X)}(X)$.

Proof. Let $Y$ be a tilting module such that we have an inclusion coker $\hookrightarrow Y$ (the existence follows from [AR91, Theorem 5.4]). Put $X:=(Q \oplus Y)_{\text {basic }}$, then there exists an exact sequence, $0 \rightarrow$ coker $\rightarrow X^{n}$, satisfying the assumptions of [KSX01, Theorem 2.8]. Hence the double centraliser property follows.

Remark 2.5. One can show that there exists a minimal basic tilting module $Y$ with the following property: any $M \in \mathcal{F}(\Delta)$ embeds into $Y^{m}$ for some $m$. Here minimal means that every other tilting module with the latter property has $Y$ as a direct summand. However, it is not clear whether there exists a minimal basic tilting module $Y$, with respect to which one has the double centraliser property. It is the case in all the examples we know, in particular in the Examples 2.7.

In general, it could happen that $X$ is already the characteristic tilting module, and the statement of Corollary 2.4 is not very useful. As an example we refer to [KK99, Example $A_{1}$ ] where the algebra $A$ is given by all $3 \times 3$ upper triangular matrices over some field $\mathbb{k}$ with the matrix idempotents $e_{1}$, 
$e_{2}, e_{3}$ and the quasi-hereditary structure given by the ordering $1<2<3$. The same algebra, but with the quasi-hereditary structure given by the reversed order (see [KK99, Example $\left.A_{2}\right]$ ) provides also an example, where $X$ is not contained in $\operatorname{Add}(Q)$, the additive category generated by $Q$. In particular, we do not have the double centralizer property with respect to $Q$.

\subsection{Double centralizer and projective-injective modules}

On the other hand, under the assumptions and notation of Proposition 2.3 we have the following nice situation, where projective-injective modules play a crucial role.

Corollary 2.6. If the injective hull of any standard module is contained in $\operatorname{Add}(Q)$, then the following holds:

$$
A \cong \operatorname{End}_{\operatorname{End}_{A}(Q)}(Q) \cong \operatorname{End}_{\operatorname{End}_{A}\left(Q_{\text {basic }}\right)}\left(Q_{\text {basic }}\right) .
$$

Proof. If the injective envelope of any standard module is contained in Add $Q$, the assumptions of [KSX01, Theorem 2.8; Theorem 2.10] are satisfied and the statement follows.

As interesting examples we have the following:

Examples 2.7. In the examples which follow we illustrate the use of Proposition 2.3 and Corollary 2.4, in particular, we explicitly describe the modules $Q$ and $X$ which appear in the double centraliser statements.

1. Let $A$ be such that $A$-mod is equivalent to an integral block of the Bernstein-Gelfand-Gelfand category $\mathcal{O}$ for some semi-simple complex Lie algebra $\mathfrak{g}$ (see [BGG76]). The algebra $A$ is equipped with the usual quasi-hereditary structure (given by the Bruhat order and the Verma modules as standard modules). In this case we have exactly one indecomposable projective-injective module, namely the projective cover $P\left(w_{0}\right)$ of the unique simple standard (or Verma) module in this block. Moreover, $A$ is Ringel self-dual ([Soe97, Theorem 5.12 and Bemerkung $2.4(3)])$. The projective cover of a tilting module is a direct sum of $P\left(w_{0}\right)$ 's. Via Ringel duality we get an inclusion

$$
i: A \hookrightarrow Q
$$

where $Q=P\left(w_{0}\right)^{n}$ for some positive integer $n$. The cokernel of this inclusion has (by Proposition 2.3) a standard (or Verma-) flag. Hence there is an exact sequence of the form

$$
0 \rightarrow A \longrightarrow Q \longrightarrow Q^{m}
$$


for some positive integer $m$. We could take $X=Q_{b a s i c}=P\left(w_{0}\right)$ and get the famous double centraliser theorem of Soergel ([Soe90], see also $\left[\mathrm{KSX} 01\right.$, Theorem 3.2]), namely $A \cong \operatorname{End}_{\operatorname{End}_{A}(X)}(X)$.

2. Let $A^{\mathfrak{p}}$ be such that $A^{\mathfrak{p}}$-mod is equivalent to an integral block of some parabolic category $\mathcal{O}^{\mathfrak{p}}$ in the sense of [RC80] (see also Section 4.2) with the usual quasi-hereditary structure. Then $A^{\mathfrak{p}}$ is Ringel-self-dual (see [Soe97] or Proposition 4.4 below). The self-dual projective modules are exactly the summands occurring in the injective hulls of standard modules ([Irv85]), they are also exactly the summands occurring in the projective cover of tilting modules. This means, we have an embedding of the form (2.2) and then an exact sequence of the form (2.3), where $Q$ is a direct sum of projective-injective modules. If we set $X=Q_{\text {basic }}$ the sum over (a system of representatives for the isomorphism classes of) all indecomposable projective-injective modules we get the double centraliser property $A^{\mathfrak{p}} \cong \operatorname{End}_{\operatorname{End}_{A^{\mathfrak{p}}}(X)}(X)$ (this is proved in [Str03b, Theorem 10.1]).

3. Let $\mathfrak{g}$ be a semisimple complex Lie algebra. Let $\mathcal{H}$ be the category of Harish-Chandra bimodules for $\mathfrak{g}$, that is the category of $\mathfrak{g}$-bimodules which are of finite length and locally finite for the adjoint action of $\mathfrak{g}$ (see for example [BG80] or [Jan83, Section 6]). The category $\mathcal{H}$ decomposes into blocks ${ }_{\lambda} \mathcal{H}_{\mu}$. A bimodule $X \in \mathcal{H}$ is contained in the block ${ }_{\lambda} \mathcal{H}_{\mu}$ if it is annihilated by $\left(\operatorname{ker} \chi_{\lambda}\right)^{n}$ from the left and by $\left(\text { ker } \chi_{\mu}\right)^{n}$ from the right for some positive integer $n$, where ker $\chi_{\lambda}$ is the annihilator of the Verma module with highest weight $\lambda$. The category ${ }_{\lambda} \mathcal{H}_{\mu}$ does not have projective objects, however, we get enough projectives (see e.g. $\left[\right.$ Jan83, 6.14]) if we consider the full subcategory ${ }_{\lambda} \mathcal{H}_{\mu}^{1}$ of ${ }_{\lambda} \mathcal{H}_{\mu}$ given by all bimodules which are annihilated by ker $\chi_{\mu}$ from the right hand side. Let $A_{\lambda}^{\mu}$ be such that $A_{\lambda}^{\mu}$-mod $\cong{ }_{\lambda} \mathcal{H}_{\mu}^{1}$, where $\lambda$ and $\mu$ are integral. Then $A_{\lambda}^{\mu}$ is standardly stratified (it is not quasi-hereditary in general) and contains a unique indecomposable projective-injective module (see [KM02, Corollary 2]). Later (Proposition 4.9) we give a new proof for the fact that $A_{\lambda}^{\mu}$ is Ringel self-dual (see [FKM00, Theorem 3] for the original argument). As in category $\mathcal{O}$, the projective cover of a tilting module is projective-injective, and hence $Q$ becomes a direct sum of copies of the unique self-dual indecomposable projective module. The injective hulls of standard modules are projective as well. Hence we could take $X=Q_{\text {basic }}$, the indecomposable projective-injective module

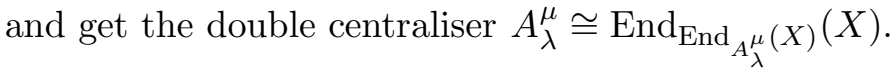

4. Let $A$ be such that $A$-mod $\cong \mathcal{O}\left(H_{c}\right)$, the category $\mathcal{O}$ for some rational Cherednik algebra $H_{c}=H_{0, c}$ as considered for example in [Gua03] 
or [GGOR03]. The projective-injective modules are exactly the summands occurring in the injective hulls of standard modules ([GGOR03, Proposition 5.21]), they are also exactly the summands occurring in the projective covers of tilting modules. Hence, $Q$ is a direct sum of projective-injectives and then we could take $X=Q_{\text {basic }}$ to be the sum over all indecomposable projective tilting modules. This is the double centraliser property from [GGOR03, Theorem 5.16].

5. Quite often there are double centraliser properties with respect to tilting modules, which do not have to be projective or injective. In the following examples the tilting module $X$ is neither projective nor injective: Let $\mathfrak{Q}$ be a finite quiver with vertices $\{1, \ldots, n\}$. Assume it is directed, that is an arrow from $i$ to $j$ exists only if $i>j$. Let $A=A(\mathfrak{Q})$ be the corresponding path algebra and $D$ be its dual extension, that is the algebra $A \otimes_{\mathbb{k}} A^{o p p}$ with the relations $\left(\operatorname{rad} A^{o p p}\right)(\operatorname{rad} A)=0$ (see e.g. [DX94]). Then $D$ is quasi-hereditary with respect to the natural order on $\{1, \ldots, n\}$. One can show that there is a double centraliser property with respect to the tilting module $X=Q_{\text {basic }}=\oplus_{i} T(i)$, where the sum runs over all sources of $\mathfrak{Q}$. It is also easy to see that $X$ is neither injective nor projective in general.

Remark 2.8. Let $A$ and $X$ be as in the examples above, then we could define

$$
\begin{aligned}
\mathbb{V}: A-\bmod & \longrightarrow \operatorname{End}_{A}(X)-\bmod \\
M & \longrightarrow \operatorname{Hom}_{A}(X, M) .
\end{aligned}
$$

The double centraliser property can be reformulated as: The functor $\mathbb{V}$ is fully faithful on projective modules, i.e. $\mathbb{V}$ induces an isomorphism

$$
\operatorname{Hom}_{A}\left(P_{1}, P_{2}\right) \cong \operatorname{Hom}_{\operatorname{End}_{A}(X)}\left(\mathbb{V} P_{1}, \mathbb{V} P_{2}\right)
$$

for all projective modules $P_{1}$ and $P_{2}$.

Another easy consequence from the definitions is the following: The functor $\mathbb{V}$ is fully faithful on tilting modules, i.e. $\mathbb{V}$ induces an isomorphism

$$
\operatorname{Hom}_{A}\left(T_{1}, T_{2}\right) \cong \operatorname{Hom}_{E_{A}(X)}\left(\mathbb{V} T_{1}, \mathbb{V} T_{2}\right)
$$

for all tilting modules $T_{1}$ and $T_{2}$.

Proof. If $\operatorname{Hom}_{A}\left(T_{1}, K\right)=0=\operatorname{Hom}_{A}\left(K, T_{2}\right)$ for any $K \in A$-mod such that $\mathbb{V} K=0$ then

$$
\operatorname{Hom}_{A}\left(T_{1}, T_{2}\right) \cong \operatorname{Hom}_{E_{A n}(X)}\left(\mathbb{V} T_{1}, \mathbb{V} T_{2}\right)
$$

since $\mathbb{V}$ is a quotient functor (see [Gab62]). All the composition factors in $K$ are annihilated by $\mathbb{V}$. On the other hand, none of the composition factors in the head of $T_{1}$ and in the socle of $T_{2}$ is annihilated by $\mathbb{V}$. This proves the statement. 


\section{Serre functors}

The aim of the present section is to develop an effective machinery to describe Serre functors for the categories appearing in the examples above, where the algebra is not given explicitly. Let $\mathcal{C}$ be a $\mathbb{k}$-linear additive category with finite dimensional homomorphism spaces. A right Serre functor is an additive endofunctor $F$ of $\mathcal{C}$ together with isomorphisms

$$
\Psi_{X, Y}: \operatorname{Hom}_{\mathcal{C}}(X, F Y) \cong \operatorname{Hom}_{\mathcal{C}}(Y, X)^{*},
$$

natural in $X$ and $Y$. Here, $*$ denotes the ordinary duality for vector spaces. Right Serre functors satisfy the following properties:

- Two right Serre functors are isomorphic (see [RVdB02, Lemma I.1.3]).

- If $\epsilon$ is an auto-equivalence of $\mathcal{C}$ and $F$ is a right Serre functor then $\epsilon F \cong F \epsilon$. (It follows directly from the definitions that $\epsilon F \epsilon^{-1}$ is a right Serre functor, hence it must be isomorphic to $F$ ).

A right Serre functor is a Serre functor if it is an auto-equivalence of $\mathcal{C}$. By general results (see [BK89]), for any finite dimensional algebra $A$ of finite global dimension, there is a Serre functor $\mathbb{S}$ for the bounded derived category $\mathcal{D}^{b}(A)$, more precisely $\mathbb{S} \cong \mathcal{L} H$, where $H=A^{*} \otimes_{A} \bullet([\mathrm{BK} 89$, Example 3.2(3)]). In the literature, the functor $H$ is often called the Nakayama functor (see e.g. [Hap88, page 37]). This is because $H \cong \operatorname{Hom}_{A}(\bullet, A)^{*}$.

Recall that for any abelian category $\mathcal{C}$ we denote by $\mathcal{D}^{b}(\mathcal{C})$ its bounded derived category. If $\mathcal{C}=A-\bmod$ we set $\mathcal{D}^{b}(A)=\mathcal{D}^{b}(\mathcal{C})$. We use the standard notation like $\mathcal{L} F, \mathcal{R} G, \mathcal{L}_{i} F, \mathcal{R}^{i} G$ etc. to denote left derived and right derived functors and their $i$-th cohomology functors. Let also $\mathcal{D}_{\text {perf }}(A)$ denote the full subcategory of $\mathcal{D}^{b}(A)$, consisting of perfect complexes (i.e. of those complexes which are quasi-isomorphic to bounded complexes of projective $A$-modules).

In order to be able to describe more explicitly the Serre functors for some of the examples mentioned above we will need effective tools to detect Serre functors. Recall that a finite-dimensional algebra, $A$, is called selfinjective provided that $A \cong A^{*}$ as left $A$-modules; and symmetric provided that $A \cong A^{*}$ as $A$-bimodules. We start with the following easy observation

Lemma 3.1. Let $A$ be a finite-dimensional self-injective algebra. Then $\mathcal{L} H$ is a Serre functor of $\mathcal{D}_{\text {perf }}(A)$, moreover, $\mathcal{L} H \cong$ ID if and only if $A$ is symmetric.

Proof. Let $\mathcal{P}^{\bullet}$ be a bounded complex of projective $A$-modules. Then we have that $\mathcal{L H P}^{\bullet}=H \mathcal{P}^{\bullet}$ is a bounded complex of injective $A$-modules by the definition of $H$. Since $A$ is self-injective we have $H \mathcal{P}^{\bullet} \in \mathcal{D}_{\text {perf }}(A)$. 
That in this case $\mathcal{L} H$ is a Serre functor is proved for example in [Gin05, Proposition 20.5.5(i)]. Finally, the last statement follows from the definition of a symmetric algebra.

Definition 3.2. Given an algebra $A$ and a projective-injective module $Q$, we call $Q$ good if the socle of $Q$ is isomorphic to the head of $Q$. (Equivalently, if $Q \cong \oplus_{\lambda \in \Lambda^{\prime}} P(\lambda)$ for some $\Lambda^{\prime} \subset \Lambda$ then $Q \cong \oplus_{\lambda \in \Lambda^{\prime}} I(\lambda)$.)

If $A$ has a duality which preserves simple modules, any projective-injective module is automatically good.

Remark 3.3. In the following we will also use double centraliser properties for the opposed algebra $A^{o p p}$. Let $I$ be a basic injective $A$-module. It is easy to see that the existence of an exact sequence of the form

$$
Q_{2} \rightarrow Q_{1} \rightarrow I \rightarrow 0
$$

for some projective-injective $A$-modules $Q_{1}, Q_{2}$, is equivalent to the requirement that $A^{\text {opp }}$ has a double centraliser property with respect to a projective-injective module. Indeed, the double centraliser property for $A^{\text {opp }}$ is equivalent to the existence of an exact sequence of the form

$$
0 \rightarrow A^{o p p} \longrightarrow X_{1}^{\prime} \longrightarrow X_{2}^{\prime}
$$

for some projective-injective modules $X_{1}^{\prime}, X_{2}^{\prime}$. Applying the usual duality $\operatorname{Hom}_{\mathbb{k}}(\bullet, \mathbb{k})$ we get an exact sequence

$$
X_{2} \rightarrow X_{1} \rightarrow I \rightarrow 0
$$

where $I$ is the injective cogenerator of $A-\bmod$ and $X_{1}, X_{2}$ are projectiveinjective.

\subsection{A characterisation of Serre functors}

The following result provides a tool to detect Serre functors:

Theorem 3.4. Let $A$ be a finite dimensional $\mathbb{k}$-algebra of finite global dimension. Assume that a basic projective-injective A-module is good and both, $A$ and $A^{\text {opp }}$, have the double centraliser property with respect to a projectiveinjective module. Let $F: A-\bmod \rightarrow A$-mod be a right exact functor. Then

$\mathcal{L} F$ is a Serre functor of $\mathcal{D}^{b}(A)$ if and only if the following conditions are satisfied:

(a) Its left derived functor $\mathcal{L} F: \mathcal{D}^{b}(A) \rightarrow \mathcal{D}^{b}(A)$ is an auto-equivalence.

(b) F maps projective A-modules to injective A-modules. 
(c) F preserves the full subcategory $\mathcal{P} \mathcal{I}$ of $A$-mod, consisting of all projective-injective modules, and the restrictions of $F$ and $H$ to $\mathcal{P} \mathcal{I}$ are isomorphic.

Proof. Let $Q$ be a good basic projective-injective $A$-module. We know that $\mathcal{D}^{b}(A)$ has a Serre functor, $\mathbb{S}$, and $\mathbb{S} \cong \mathcal{L} H$, where $H=A^{*} \otimes_{A} \bullet$. By definition, $H$ satisfies (a) and (b) and preserves $\mathcal{P} \mathcal{I}$, because $Q$ is good. Hence $H$ satisfies (c).

Now let $F: A$-mod $\rightarrow A$-mod be a right exact functor, satisfying (a)(c). We claim that $F$ and $H$ are isomorphic when restricted to the category of injective $A$-modules. Indeed, the double centraliser property for $A^{\text {opp }}$ gives us an exact sequence,

$$
X_{2} \rightarrow X_{1} \rightarrow I \rightarrow 0
$$

where $I$ is the injective cogenerator of $A-\bmod$ and $X_{1}, X_{2} \in \mathcal{P I}$ (see Remark 3.3). Let $\psi: F \rightarrow H$ be the isomorphism, given by (c). Applying $F$ and $H$ to (3.5) and using (c) we obtain the following diagram with exact rows, where the square on the left hand side commutes, inducing an isomorphism, $\psi_{I}$, as indicated:

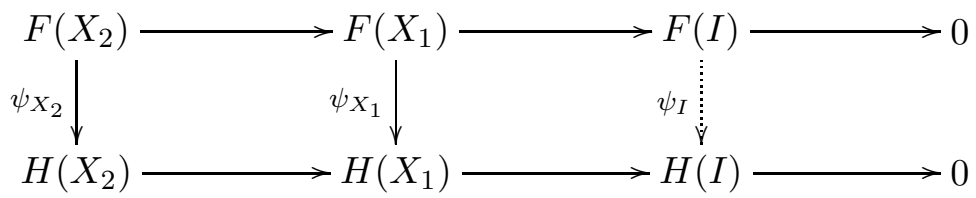

By standard arguments, it defines an isomorphism of functors, $F \cong H$, when restricted to the full additive category of injective $A$-modules. Since $\mathcal{L} F$ is an auto-equivalence, we have $\mathcal{L} F \mathbb{S} \cong \mathbb{S} \mathcal{L} F$. As projectives are acyclic for right exact functors, we get an isomorphism, $\mathcal{L} F H \cong \mathbb{S} F$, when restricted to the full additive subcategory given by projectives. Taking the 0 -th homology we get an isomorphism of functors

$$
F H \cong H F
$$

when restricted to the full additive category of projective $A$-modules. Since the functors $F$ and $H$ are right exact, we only have to deduce that $F \cong H$ on the category of projectives. We already know that $F$ and $H$ are invertible on $\mathcal{P} \mathcal{I}$, hence we can fix isomorphisms $\alpha: \operatorname{End}_{A}(Q) \cong \operatorname{End}_{A}(F Q)$ and $\beta: \operatorname{End}_{A}(Q) \cong \operatorname{End}_{A}(H Q)$. When restricted to $\mathcal{P} \mathcal{I}$, we have $F \cong \mathrm{ID}^{\alpha}$ and $H \cong \mathrm{ID}^{\beta}$, where $\mathrm{ID}^{\alpha}$ and $\mathrm{ID}^{\beta}$ denote the identity functors, but with the $\operatorname{End}_{A}(Q)$-action twisted by $\alpha$ or $\beta$ respectively. Since both, $F$ and $H$, are right exact, they uniquely extend to functors on $\bmod -\operatorname{End}_{A}(Q)$, the latter being realized as the full subcategory $\mathcal{C}$ of $A$-mod given by all modules, having a presentation of the form (3.5) (see [Aus74, Section 5]). From the 
explicit description above, we obtain that both $F$ and $H$ are invertible as endofunctors of $\mathcal{C}$. As both, $H$ and $F$, map projectives to injectives and $F \cong H$ on injectives we get, together with (3.6), isomorphisms of functors $F^{2} \cong H F \cong F H$ when restricted to the full additive category of projective $A$-modules. This gives then rise to an isomorphism, $F \cong H$, since $F$ is invertible on $\mathcal{C}$. So, we are done.

Proposition 3.5. Let $A$ be a finite dimensional $\mathbb{k}$-algebra of finite global dimension. Assume there is a good basic projective-injective module $Q$ and set $B=\operatorname{End}_{A}(Q)$. Then the algebra $B$ is symmetric if and only if the restriction of the Serre functor for $\mathcal{D}^{b}(A)$ to $\mathcal{P} \mathcal{I}$ is the identity functor.

Proof. Let $\mathbb{S}$ be the Serre functor for $\mathcal{D}^{b}(A)$. Then $\mathbb{S}$ obviously preserves $\mathcal{P} \mathcal{I}$, because $Q$ is good, and hence it also preserves the (homotopy) category of bounded complexes of projective-injective $A$-modules. Moreover, it induces a Serre functor on this category. By [Aus74, Section 5], the latter one is equivalent to the category $\mathcal{D}_{\text {perf }}(B)$. The statement now follows from Lemma 3.1.

\subsection{Serre functors via partial coapproximation}

In this subsection we want to show that double centraliser properties with respect to projective-injective modules quite often make it possible to describe the Serre functor in terms of partial coapproximations.

For the remaining section let $A$ be a finite dimensional algebra of finite global dimension. Let $Q \in A$-mod be a projective module. For any module $M$ let $M_{Q}$ be the trace of $Q$ in $M$ (ie. $M_{Q}$ is the smallest submodule of $M$ such that $\left.\operatorname{Hom}_{A}\left(Q, M / M_{Q}\right)=0\right)$. Dually let $M^{Q}$ be the smallest quotient of $M$ such that $\operatorname{Hom}_{A}(Q, M)=\operatorname{Hom}_{A}\left(Q, M^{Q}\right)$.

Associated with $Q$, there is a right exact functor $\operatorname{Coapp}_{Q}: A-\bmod \rightarrow$ $A$-mod called the partial coapproximation with respect to $Q$ (for details we refer for example to $[\mathrm{KM} 05,2.5])$. It sends a projective module $P$ to $P_{Q}$. Note that if $f: P \rightarrow P^{\prime}$ is a morphism between projective modules, then it induces a morphism, $\operatorname{Coapp}_{Q}(f):$ Coapp $P \rightarrow \operatorname{Coapp}\left(P^{\prime}\right)$. These assignments can be extended uniquely to a right exact endofunctor $\operatorname{Coapp}_{Q}$ of $A$-mod. For an arbitrary module $M \in A$-mod, the module Coapp ${ }_{Q} M$ can be constructed in the following way: We choose a short exact sequence $K \hookrightarrow P \rightarrow M$, where $P$ is projective. Then

$$
\operatorname{Coapp}_{Q} M \cong\left(P / K_{Q}\right)_{Q},
$$

in other words $\operatorname{Coapp}_{Q} M$ is obtained from $M$ by first maximally extending $M$ using simple modules, which do not occur in the top of $Q$, and afterwards deleting all occurrences of such modules in the top part. 
Lemma 3.6. Let $A$ be a finite dimensional $\mathbb{k}$-algebra. Assume, $A^{\text {opp }}$ has the double centraliser property with respect to a projective-injective module. Let $Q$ be a basic projective-injective A-module. Let $\lambda \in \Lambda$. Then the following holds: If $P(\lambda)_{Q} \cong I(\lambda)^{Q}$ then $\left(\operatorname{Coapp}_{Q}\right)^{2}(P(\lambda)) \cong I(\lambda)$.

Proof of Lemma 3.6. We have

$$
\begin{aligned}
\left(\operatorname{Coapp}_{Q}\right)^{2}(P(\lambda)) & \cong \operatorname{Coapp}_{Q}\left(P(\lambda)_{Q}\right) \\
& \cong \operatorname{Coapp}_{Q}\left(I(\lambda)^{Q}\right) \\
& \cong I(\lambda)
\end{aligned}
$$

Here, only the last isomorphism needs some explanation. If $P$ is the projective cover of $I(\lambda)^{Q}$ then the natural surjection from $I(\lambda)$ onto $I(\lambda)^{Q}$ lifts to a map, $f: P \rightarrow I(\lambda)$. From the definition of $I(\lambda)^{Q}$ and (3.2) it follows that $f$ is surjective. The double centraliser property for $A^{o p p}$ (see Remark 3.3) also implies that any composition factor in the head of the kernel of $f$ is not annihilated by $\operatorname{Hom}_{A}(Q, \bullet)$. Hence the desired isomorphism follows.

The following theorem describes a situation, where the double centraliser property with respect to a basic projective-injective module $Q$, the description of the Serre functor via partial coapproximation, and the symmetry of the endomorphism ring of $Q$ are nicely connected. Later on we will see that this setup applies to all the different versions of category $\mathcal{O}$ mentioned in the Examples 2.7.

Theorem 3.7. Let $A$ be a finite dimensional $\mathbb{k}$-algebra of finite global dimension. Let $Q$ be a basic projective-injective A-module. Assume, $Q$ is good and both, $A$ and $A^{\text {opp }}$ have the double centraliser property with respect to some projective-injective module. Consider the functors $\mathbb{V}=\operatorname{Hom}_{A}(Q, \bullet)$ :

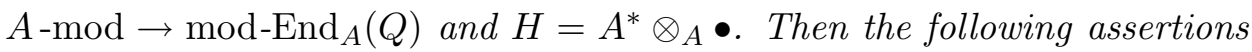
are equivalent:

(i) $\mathbb{V} \cong \mathbb{V} H$,

(ii) $H \cong\left(\operatorname{Coapp}_{Q}\right)^{2}$,

(iii) $\operatorname{End}_{A}(Q)$ is symmetric.

In either of these cases, the Serre functor for $\mathcal{D}(A)^{b}$ is $\mathcal{L}\left(\left(\operatorname{Coapp}_{Q}\right)^{2}\right)$.

Proof. Obviously, if (ii) holds then $\mathcal{L}\left(\left(\operatorname{Coapp}_{Q}\right)^{2}\right)$ is the Serre functor for $\mathcal{D}^{b}(A)$. It is left to show that the three cases are equivalent.

(i) $\Rightarrow$ (ii) : Let us assume $\mathbb{V} \cong \mathbb{V} H$. Let $P$ be a projective module. By the 
assumed double centraliser property for $A$ and $A^{\text {opp }}$ (see Remark 3.3) we have natural isomorphisms

$$
\begin{aligned}
\operatorname{Hom}_{A}(P, P) & \cong \operatorname{Hom}_{\operatorname{End}_{A}(Q)}(\mathbb{V} P, \mathbb{V} P) \\
& \cong \operatorname{Hom}_{\operatorname{End}_{A}(Q)}(\mathbb{V} H P, \mathbb{V} P) \\
& \cong \operatorname{Hom}_{A}(H P, P)
\end{aligned}
$$

(for the last isomorphism we refer to the proof of (2.4)). The identity map in $\operatorname{End}_{A}(P)$ gives rise to a natural morphism, $H P \rightarrow P$, identifying $(H P)^{Q}$ and $P_{Q}$. Since $H$ maps the projective cover of any simple module to its injective hull, we are in the situation of Lemma 3.6. In particular, $\left(\operatorname{Coapp}_{Q}\right)^{2}$ sends an indecomposable projective module to the corresponding indecomposable injective module. Let $G$ be the right adjoint functor to Coapp $_{Q}$ (this is the functor of partial approximation with respect to $Q$, see $[\mathrm{KM} 05,2.5])$. We have the adjunction morphism ID $\rightarrow G^{2}\left(\operatorname{Coapp}_{Q}\right)^{2}$ which we know is an isomorphism on projective-injective modules. From the double centraliser property we get that this adjunction morphism is injective on all projective modules. Since $\left(\operatorname{Coapp}_{Q}\right)^{2} P$ is isomorphic to the corresponding injective module, we have $G^{2}\left(\operatorname{Coapp}_{Q}\right)^{2} \cong P$. In particular, $G^{2}\left(\operatorname{Coapp}_{Q}\right)^{2} \cong$ ID when restricted to the additive subcategory given by projective modules. Dually, $\left(\operatorname{Coapp}_{Q}\right)^{2} G^{2} \cong \mathrm{ID}$ when restricted to the additive category given by injective modules. Since $A$ has finite global dimension, $\mathcal{L}\left(\left(\text { Coapp }_{Q}\right)^{2}\right)$ defines an auto-equivalence of the derived category $\mathcal{D}^{b}(A)$ with inverse $\mathcal{R} G^{2}$. From our assumption we have $\mathbb{V}\left(\operatorname{Coapp}_{Q}\right)^{2} \cong \mathbb{V} \cong \mathbb{V} H$. Therefore, $\left(\operatorname{Coapp}_{Q}\right)^{2} \cong$ $H$ on the additive subcategory given by all projective-injective modules. Hence $\left(\operatorname{Coapp}_{Q}\right)^{2}$ satisfies the assumptions of Theorem 3.4. It follows in particular, $H \cong\left(\operatorname{Coapp}_{Q}\right)^{2}$.

(ii) $\Rightarrow$ (iii) : The definition of $\operatorname{Coapp}_{Q}$ implies that it induces the identity functor on the category of projective-injective $A$-modules. Hence $\operatorname{End}_{A}(Q)$ is symmetric by Proposition 3.5.

(iii) $\Rightarrow$ (i) : We assume that $B=\operatorname{End}_{A}(Q)$ is symmetric. From Lemma 3.1 we have that the Serre functor of $\mathcal{D}_{\text {perf }}(B)$ is isomorphic to the identity functor. On the other hand, the Serre functor of $\mathcal{D}^{b}(A)$ induces a Serre functor on the category of bounded complexes of projective-injective $A$ modules. (Note that this category is preserved by the Serre functor, since $Q$ was assumed to be good.) Altogether, when restricted to the category of projective-injective modules, the functor $H$ is isomorphic to the identity functor. This provides the following sequence of natural isomorphisms for any projective $A$-module $P$ :

$$
\begin{aligned}
\mathbb{V} H P & \cong \operatorname{Hom}_{A}(Q, H P) \\
& \cong \operatorname{Hom}_{A}(H Q, H P) \\
& \cong \operatorname{Hom}_{A}(Q, P) \\
& \cong \mathbb{V} P .
\end{aligned}
$$


(For the penultimate isomorphism we used that $H$ defines an auto-equivalence of $\mathcal{D}^{b}(A)$, hence it is in particular fully faithful on projectives.) Thus we get an isomorphism of functors $\mathbb{V} H \cong \mathbb{V}$ when restricted to the category of projective modules. Since the involved functors are right exact, the isomorphism extends to an isomorphism of functors $\mathbb{V} H \rightarrow \mathbb{V}$.

\section{Applications}

\subsection{Bernstein-Gelfand-Gelfand category $\mathcal{O}$}

Let $\mathfrak{g}$ be a semisimple complex Lie algebra with a fixed Borel subalgebra $\mathfrak{b}$ containing the fixed Cartan subalgebra $\mathfrak{h}$. Let $\mathcal{O}$ be the corresponding BGG-category (see [BGG76]). Let $W$ denote the Weyl group of $\mathfrak{g}$ with longest element $w_{0}$. For any weight $\lambda \in \mathfrak{h}^{*}$ let $W_{\lambda}$ be the stabiliser $W_{\lambda}=$ $\{w \in W \mid w \cdot \lambda=\lambda\}$, where $w \cdot \lambda=w(\lambda+\rho)-\rho$ and $\rho$ is the half-sum of positive roots. For $\mu \in \mathfrak{h}^{*}$ let $\Delta(\mu)$ be the Verma module with highest weight $\mu$. For $\lambda \in \mathfrak{h}^{*}$, a dominant and integral weight, we consider the block $\mathcal{O}_{\lambda}$, containing the Verma modules $\Delta(\mu)$, where $\mu \in W \cdot \lambda$. Let $L(\mu)$ be the simple quotient of $\Delta(\mu)$ and $P(\mu)$ its projective cover. For any $w \in W$, there is a twisting functor $T_{w}: \mathcal{O} \rightarrow \mathcal{O}$ (given by tensoring with some "semiregular bimodule"), see [AL03], [KM05] or [AS03] for a precise definition. Let $\mathrm{d}$ be the duality on $\mathcal{O}$. We denote by $G_{w}$ the right adjoint functor of $T_{w}$. We have $G_{w} \cong \mathrm{d} T_{w} \mathrm{~d}$ (see [AS03, Section 4]).

If $\lambda$ is regular, and $s$ is a simple reflection, we denote by $C_{s}$ Irving's shuffling functor defined as taking the cokernel of the adjunction morphism between the identity functor and the translation $\theta_{s}$ "through the $s$-wall" ([GJ81, Section 3]). Let $w_{0}=s_{i_{1}} s_{i_{2}} \cdots s_{i_{r}}$ be a reduced expression, then we define $C_{w_{0}}=C_{s_{i_{r}}} C_{s_{i_{r-1}}} \cdots C_{s_{i_{1}}}$. Up to isomorphism, this does not depend on the chosen reduced expression (see e.g. [MS05, Lemma 5.10]).

Proposition 4.1. Let $A=A_{\lambda}$ such that $A_{\lambda}-\bmod \cong \mathcal{O}_{\lambda}$ for some integral block $\mathcal{O}_{\lambda}$.

(1) The functor $\mathcal{L}\left(T_{w_{0}}\right)^{2}: \mathcal{D}^{b}\left(\mathcal{O}_{\lambda}\right) \rightarrow \mathcal{D}^{b}\left(\mathcal{O}_{\lambda}\right)$ is a Serre functor.

(2) If $\lambda$ is regular, then $\mathcal{L}\left(C_{w_{0}}\right)^{2}: \mathcal{D}^{b}\left(\mathcal{O}_{\lambda}\right) \rightarrow \mathcal{D}^{b}\left(\mathcal{O}_{\lambda}\right)$ is a Serre functor. In particular, $\mathcal{L}\left(T_{w_{0}}\right)^{2} \cong \mathcal{L}\left(C_{w_{0}}\right)^{2}$.

Proof. We want to verify the assumptions of Theorem 3.4 for $A=A_{\lambda}$ and $F=T_{w_{0}}^{2}$ considered as an endofunctor of $A$-mod.

Because of the existence of a duality on $A$ we have $A \cong A^{\text {opp }}$ and, as we have already mentioned in the introduction, $A$ has a double centraliser property with respect to the good basic projective-injective module $P\left(w_{0} \cdot \lambda\right)$ (see [Soe90, Struktursatz]). If $\lambda$ is regular, the endomorphism algebra of the 
latter is the coinvariant algebra associated with $W$. If $\lambda$ is singular then this endomorphism ring is isomorphic to the subalgebra of $W_{\lambda}$-invariants in the coinvariant algebra ([Soe90, Endomorphismensatz]). In any case, the resulting algebra is symmetric. Consider now $\left(T_{w_{0}}\right)^{2}: \mathcal{O}_{\lambda} \rightarrow \mathcal{O}_{\lambda}$. This functor is both right exact and additive by definition. It's derived functor defines a self-equivalence of $\mathcal{D}^{b}\left(\mathcal{O}_{\lambda}\right)$ by [AS03, Corollary 4.2] for the regular case; the singular case follows by translation, since twisting functors commute naturally with translation functors ([AS03, Theorem 3.2]). Hence the assumption (a) of Theorem 3.4 is satisfied.

From [AS03, (2.3) and Theorem 2.3] we have

$$
F(P(\lambda))=F(\Delta(\lambda)) \cong \nabla(\lambda) \cong I(\lambda)
$$

if $\lambda$ is regular. By [AS03, Theorem 3.2], $F$ commutes with projective functors. Applying projective functors to (4.1) gives $F(P(\mu))=I(\mu)$ for any $\mu \in W \cdot \lambda$. Hence, the assumption (b) of Theorem 3.4 is satisfied. It is left to verify the assumption (c) of Theorem 3.4.

Since the endomorphism ring of $P\left(w_{0} \cdot \lambda\right)$ is symmetric, by Proposition 3.5 it is left to check that $T_{w_{0}}$ is isomorphic to the identity functor when restricted to the category of projective-injective modules. By [KM05, Theorem 4], there is a natural transformation, $T_{w_{0}} \rightarrow$ ID, which is an isomorphism, when restricted to projective-injective modules ([AS03, Proposition 5.4]). In particular, the assumption (c) of Theorem 3.4 is satisfied. Theorem 3.4 therefore implies that $\mathcal{L}\left(T_{w_{0}}\right)^{2}$ is a Serre functor of $\mathcal{D}^{b}\left(\mathcal{O}_{\lambda}\right)$. The first part of the proposition follows.

Let now $\lambda$ be dominant, integral and regular. We again want to apply Theorem 3.4. The functor $F=\left(C_{w_{0}}\right)^{2}: \mathcal{O}_{\lambda} \rightarrow \mathcal{O}_{\lambda}$ is both right exact and additive by definition. Its derived functor defines a self-equivalence of $\mathcal{D}^{b}\left(\mathcal{O}_{\lambda}\right)$ by [MS05, Theorem 5.7]. That $F(P(\mu))=I(\mu)$ for any $\mu \in W \cdot \lambda$ follows inductively from [Irv93, Proposition 3.1], [MS05, Theorem 5.7, Lemma 5.2 and Proposition 5.3]. Since $\operatorname{End}_{\mathfrak{g}}\left(P\left(w_{0} \cdot \lambda\right)\right)$ is symmetric, it is, by Proposition 3.5 , left to check that $F$ is isomorphic to the identity functor when restricted to the category of projective-injective modules. That $F$ preserves projective-injective modules follows from [Irv93, Theorem 4.1(1)]. From [Soe90, Section 2.4] it follows that $F$ commutes with the action of the centre of $A$, which, because of the double centraliser and commutativity of $\operatorname{End}_{\mathfrak{g}}\left(P\left(w_{0} \cdot \lambda\right)\right)$, is in fact $\operatorname{End}_{\mathfrak{g}}\left(P\left(w_{0} \cdot \lambda\right)\right)$. This implies that $F$, restricted to the category of projective-injective modules, is isomorphic to the identity functor. Theorem 3.4 now implies that $\mathcal{L}\left(C_{w_{0}}\right)^{2}$ is a Serre functor of $\mathcal{D}^{b}\left(\mathcal{O}_{\lambda}\right)$. From the uniqueness of Serre functors we get in particular $\mathcal{L}\left(T_{w_{0}}\right)^{2} \cong \mathcal{L}\left(C_{w_{0}}\right)^{2}$.

We obtain the following surprising consequence: 
Corollary 4.2. Let $\lambda$ be an integral, dominant and regular weight. Considered as endofunctors of $\mathcal{O}_{\lambda}$, there is an isomorphism of functors $\left(C_{w_{0}}\right)^{2} \cong$ $\left(T_{w_{0}}\right)^{2}$. In particular $\left(C_{w_{0}}\right)^{2}$ commutes with projective functors.

Proof. The functors are isomorphic when restricted to the additive category of projective modules, since they both give rise to a Serre functor. On the other hand, they are both right exact and $\mathcal{O}_{\lambda}$ has finite global dimension. Therefore, the isomorphism extends uniquely to the whole category $\mathcal{O}_{\lambda}$. Twisting functors commute with projective functors (see [AS03, Section 3]), hence $\left(C_{w_{0}}\right)^{2}$ commutes with projective functors as well.

Remark 4.3. We would like to draw the reader's attention to the following observations concerning the principal block $\mathcal{O}_{0}$ of $\mathcal{O}$ :

1.) The functor $\mathrm{d} T_{w_{0}}^{2} \mathrm{~d}$ is exactly Enright's completion functor, see e.g. [Jos82]. This follows from [KM05, Section 3].

2.) Considered as an endofunctor of $\mathcal{O}_{0}$, the functor $C_{w_{0}}$ does not commute with the action of the centre of the universal enveloping algebra of $\mathfrak{g}$ (or with the centre of $\mathcal{O}_{0}$ ) and does not commute with translation functors even if $\mathfrak{g}=\mathfrak{s l}_{2}$ (whereas $T_{w_{0}}$ does, see [AS03, Section 3]). This is because $C_{w_{0}}$ twists the action of the centre by $w_{0}$ (this follows from [Soe90, Section 2.4]). This means, however, that $C_{y^{-1}} C_{y}$ commutes with the action of the centre of the category for any $y \in W$ (however, not necessarily with projective functors).

3.) Since $\left(C_{w_{0}}\right)^{2}$ induces the identity on the category of injective modules, it follows that $\left(C_{w_{0}}\right)^{4} \cong\left(C_{w_{0}}\right)^{2}$. It is easy to see that already $T_{w_{0}}^{3} \cong$ $T_{w_{0}}^{2}$. Moreover, the functors $C_{w_{0}},\left(C_{w_{0}}\right)^{2}$, and $C_{w_{0}}^{3}$ are pairwise nonisomorphic; and the functors $T_{w_{0}}, T_{w_{0}}^{2}$ are not isomorphic.

4.) If $w_{0}=s_{1} \ldots s_{k}$ is a reduced decomposition, then

$$
\begin{aligned}
& \mathcal{L}\left(C_{w_{0}}\right)^{2} \cong\left(\mathcal{L} C_{s_{1}} \mathcal{L} C_{s_{2}} \cdots \mathcal{L} C_{s_{t}}\right)\left(\mathcal{L} C_{s_{1}} \mathcal{L} C_{s_{2}} \cdots \mathcal{L} C_{s_{t}}\right) \\
& \mathcal{L}\left(T_{w_{0}}\right)^{2} \cong\left(\mathcal{L} T_{s_{1}} \mathcal{L} T_{s_{2}} \cdots \mathcal{L} T_{s_{t}}\right)\left(\mathcal{L} T_{s_{1}} \mathcal{L} T_{s_{2}} \cdots \mathcal{L} T_{s_{t}}\right)
\end{aligned}
$$

The first isomorphism follows for example from [Irv93, Proposition 3.1] by standard arguments. The second follows directly from [AS03, Theorem 2.2, Theorem 2.3 and Theorem 3.2].

5.) The Serre functor $\mathbb{S}$ for $\mathcal{D}^{b}\left(\mathcal{O}_{0}\right)$ satisfies $\mathbb{S}^{k} \neq \mathbb{S}^{l}$ for all $k \neq l$. Indeed, from [AS03, Corollary 6.2] it follows that $\mathbb{S}^{k} L(0) \cong L(0)\left[k 2 l\left(w_{0}\right)\right]$. From $\left[\right.$ AS03, Corollary 6.2] it also follows that $\mathbb{S}^{k} \neq[l]$ for any $k, l$ because $\mathbb{S} P\left(w_{0} \cdot 0\right) \cong P\left(w_{0} \cdot 0\right)$. 
6.) The braid group acts on $\mathcal{D}^{b}\left(\mathcal{O}_{0}\right)$ via the auto-equivalences $\mathcal{L} C_{s}$ and via the auto-equivalences $\mathcal{L} T_{s}$. Since the Serre functor commutes with autoequivalences, it is natural to expect that it should correspond to a central element in the Braid group. In fact, $s_{1} \ldots s_{k} s_{1} \ldots s_{k}$ (see notation above) generates the centre of the Braid group $B_{n}, n \geq 3$, see for example [Bir74, Corollary 1.8.4].

\subsection{The parabolic category $\mathcal{O}$ in the sense of Rocha-Caridi}

Our next task is to describe the Serre functor for the bounded derived category associated with the principal block of a parabolic category $\mathcal{O}$ in the sense of [RC80]. The situation here is much more complicated, since there are in general non-isomorphic indecomposable projective-injective modules in the same block and we do not yet know if the endomorphism ring of a basic projective-injective module is symmetric. However, the knowledge of the Serre functor for the bounded derived category of $\mathcal{O}_{0}$ turns out to be extremely useful to determine the Serre functor for the parabolic situation.

Let $\mathfrak{p} \supset \mathfrak{b}$ be a parabolic subalgebra of $\mathfrak{g}$ with corresponding Weyl group $W_{\mathfrak{p}} \subset W$. Let $w_{0}^{\mathfrak{p}}$ be the longest element in $W_{\mathfrak{p}}$. For any integral dominant weight $\lambda$ let $\mathcal{O}_{\lambda}^{\mathfrak{p}}$ be the full subcategory of $\mathcal{O}_{\lambda}$ given by locally $\mathfrak{p}$-finite objects. This category was introduced in [RC80]. For any $w \in W$ let $\Delta^{\mathfrak{p}}(w \cdot \lambda)$ denote the corresponding parabolic Verma module with highest weight $w \cdot \lambda$, i.e. the maximal quotient, contained in $\mathcal{O}_{\lambda}^{\mathfrak{p}}$, of the Verma module $\Delta(w \cdot \lambda) \in \mathcal{O}_{\lambda}$. Note that $\Delta^{\mathfrak{p}}(w \cdot \lambda) \neq 0$ if and only if $w$ is a shortest coset representative in $W_{\mathfrak{p}} \backslash W$.

Let from now on $\lambda$ be dominant, integral and regular. Since any object in $\mathcal{D}^{b}\left(\mathcal{O}_{\lambda}\right)$ for which all cohomology objects are contained in $\mathcal{O}_{\lambda}^{\mathfrak{p}}$ is quasiisomorphic to some complex of objects from $\mathcal{O}_{\lambda}^{\mathfrak{p}}$ (see the proof of [KS94, Proposition 1.7.11]), $\mathcal{D}^{b}\left(\mathcal{O}_{\lambda}^{\mathfrak{p}}\right)$ embeds as a full triangulated subcategory in $\mathcal{D}^{b}\left(\mathcal{O}_{\lambda}\right)$. Note that translations through walls preserve the parabolic subcategory. We may therefore consider the restriction of $\mathcal{L} C_{w_{0}}$ to this subcategory as well as to the subcategory $\mathcal{O}_{\lambda}^{\mathfrak{p}}$ (considered as a subcategory of $\mathcal{D}^{b}\left(\mathcal{O}_{\lambda}^{\mathfrak{p}}\right)$ ). We get the following result:

Proposition 4.4. For any integral dominant and regular weight $\lambda$ we have:

(1) The functor $\mathcal{L}\left(C_{w_{0}}\right)\left[-l\left(w_{0}^{\mathfrak{p}}\right)\right]$ maps parabolic Verma modules to parabolic dual Verma modules. More precisely

$$
\mathcal{L}\left(C_{w_{0}}\right)\left[-l\left(w_{0}^{\mathfrak{p}}\right)\right] \Delta^{\mathfrak{p}}(w \cdot \lambda) \cong \mathrm{d} \Delta^{\mathfrak{p}}\left(w_{0}^{\mathfrak{p}} w w_{0} \cdot \lambda\right)
$$

for any parabolic Verma module $\Delta^{\mathfrak{p}}(w \cdot \lambda) \in \mathcal{O}_{\lambda}^{\mathfrak{p}}$.

(2) The category $\mathcal{O}_{\lambda}^{\mathfrak{p}}$ is Ringel self-dual. 
(3) The functor $\mathcal{L}\left(C_{w_{0}}\right)^{2}\left[-2 l\left(w_{0}^{\mathfrak{p}}\right)\right]$ maps projectives in $\mathcal{O}^{\mathfrak{p}}$ to injectives in $\mathcal{O}^{\mathfrak{p}}$.

(4) The functor $\mathcal{L}\left(C_{w_{0}}\right)^{2}\left[-2 l\left(w_{0}^{\mathfrak{p}}\right)\right]$ is a Serre functor for $\mathcal{D}^{b}\left(\mathcal{O}_{\lambda}^{\mathfrak{p}}\right)$.

Proof. We first check that $w_{0}^{\mathfrak{p}} w w_{0}$ is indeed a shortest coset representative, if so is $w$. Let $s \in W_{\mathfrak{p}}$ be a simple reflection. Then

$$
\begin{aligned}
& l\left(s w_{0}^{\mathfrak{p}} w w_{0}\right)=l\left(w_{0}\right)-l\left(s w_{0}^{\mathfrak{p}} w\right)=l\left(w_{0}\right)-l\left(s w_{0}^{\mathfrak{p}}\right)-l(w)= \\
& =l\left(w_{0}\right)-l\left(w_{0}^{\mathfrak{p}}\right)+1-l(w)=l\left(w_{0}\right)-l\left(w_{0}^{\mathfrak{p}} w\right)+1=l\left(w_{0}^{\mathfrak{p}} w w_{0}\right)+1 .
\end{aligned}
$$

Let $\Delta^{\mathfrak{p}}(w \cdot \lambda)$ be a parabolic Verma module in $\mathcal{O}_{\lambda}^{\mathfrak{p}}$. From [Lep77, Section 4] we have a finite resolution, $P_{\bullet}^{w}$, of $\Delta^{\mathfrak{p}}(w \cdot \lambda)$ by Verma modules, where

$$
P_{i}^{w}=\bigoplus_{y \in W_{\mathfrak{p}}, l(y)=i} \Delta(y w \cdot \lambda)
$$

The involved maps are clear up to scalars, for the exact normalisation we refer to [Lep77, Lemma 4.1].

For a simple reflection, $s$, the definition of $C_{s}$ implies $C_{s} \Delta(x \cdot \lambda) \cong$ $\Delta(x s \cdot \lambda)$ if $x s>x$, and $C_{s} \nabla(x \cdot \lambda) \cong \nabla(x s \cdot \lambda)$ if $x s<x$. Therefore $C_{w_{0}} \Delta(x \cdot \lambda) \cong \nabla\left(x w_{0} \cdot \lambda\right)$, which implies that $C_{w_{0}}$ is exact on the category of modules with Verma flag. This gives $\mathcal{R} C_{w_{0}} \Delta^{\mathfrak{p}}(w \cdot \lambda) \cong C_{w_{0}}\left(P_{\bullet}^{w}\right)$. Dually, $\mathrm{d} P_{\bullet}^{w_{0}^{\mathfrak{p}} w w_{0}}$ is a coresolution of $\mathrm{d} \Delta^{\mathfrak{p}}\left(w_{0}^{\mathfrak{p}} w w_{0} \cdot \lambda\right)$. On the other hand, applying $C_{w_{0}}$ to the resolution $P_{\bullet}^{w}$ gives a complex, $Q_{\bullet}$, where

$$
Q_{i}=\bigoplus_{y \in W_{\mathfrak{p}}, l(y)=i} \mathrm{~d} \Delta\left(y w w_{0} \cdot \lambda\right)=\bigoplus_{y \in W_{\mathfrak{p}}, l(y)=i} \nabla\left(y w w_{0} \cdot \lambda\right)
$$

The maps in this complex satisfy the dual version of [Lep77, Lemma 4.1]. Hence $\mathrm{d} P_{\bullet}^{w_{0}^{\mathfrak{p}} w w_{0}} \cong Q_{\bullet}\left[-l\left(w_{0}^{\mathfrak{p}}\right)\right]$. The formula (4.2) follows.

Let now $F=\mathcal{L}_{l\left(w_{0}^{p}\right)} C_{w_{0}}$. We claim that $F$, restricted to $\mathcal{O}_{\lambda}^{\mathfrak{p}}$, is right exact. Note that the formulas above imply that $\mathcal{L}_{i} C_{w_{0}} M=0$ for any $M \in \mathcal{F}\left(\Delta^{\mathfrak{p}}\right)$ and $i \neq l\left(w_{0}^{\mathfrak{p}}\right)$, in particular, for $M \in \mathcal{O}_{\lambda}^{\mathfrak{p}}$ projective. Let $M \in \mathcal{O}_{\lambda}^{\mathfrak{p}}$ be arbitrary. Choose a short exact sequence $K \hookrightarrow P \rightarrow M$, where $P \in \mathcal{O}_{\lambda}^{\mathfrak{p}}$ is projective. Since the global dimension of $A_{\lambda}^{\mathfrak{p}}$ is finite, one obtains $\mathcal{L}_{i} C_{w_{0}} M=$ 0 for all $M$ and all $i<l\left(w_{0}^{\mathfrak{p}}\right)$ by induction. Therefore, $F$ is right exact. It is known (see e.g. [MS05, Lemma 5.1, Lemma 5.2]) that $G=\mathcal{R}^{l\left(w_{0}^{\mathfrak{p}}\right)}\left(\mathrm{d} C_{w_{0}} \mathrm{~d}\right.$ ) is the right adjoint functor of $F$. From the formula (4.2) it follows that $F$ defines an equivalence $\mathcal{F}\left(\Delta^{\mathfrak{p}}\right) \cong \mathcal{F}\left(\nabla^{\mathfrak{p}}\right)$ with inverse $G$. Proposition 2.2 implies that $\mathcal{O}_{\lambda}^{\mathfrak{p}}$ is Ringel self-dual. Applying Proposition 2.2 twice, we get that the functor $\mathcal{L}\left(C_{w_{0}}\right)^{2}\left[-2 l\left(w_{0}^{\mathfrak{p}}\right)\right]$ maps projective modules to injective modules. To prove that $\mathcal{L}\left(C_{w_{0}}\right)^{2}\left[-2 l\left(w_{0}^{\mathfrak{p}}\right)\right]$ is a Serre functor we only have to verify the last assumption of Theorem 3.4. This is not completely trivial. Instead of applying again Theorem 3.4 we will give an alternative argument after the following lemma. 
Let $\lambda$ still be dominant, integral and regular. Let $\mathfrak{i}: \mathcal{O}_{\lambda}^{\mathfrak{p}} \rightarrow \mathcal{O}_{\lambda}$ denote the exact inclusion functor, let $\mathrm{Z}: \mathcal{O}_{\lambda} \rightarrow \mathcal{O}_{\lambda}^{\mathfrak{p}}$ be its left adjoint and $\mathrm{Z}: \mathcal{O}_{\lambda} \rightarrow \mathcal{O}_{\lambda}^{\mathfrak{p}}$ be the right adjoint to $\mathfrak{i}$ (i.e. $\mathrm{Z}$ is the Zuckerman functor of taking the maximal quotient in $\mathcal{O}_{\lambda}^{\mathfrak{p}}, \hat{\mathrm{Z}} \cong \mathrm{d} \mathrm{Z} \mathrm{d}$ ). To proceed we will need the following result from folklore:

Lemma 4.5. There are isomorphisms of functors:

$$
\begin{aligned}
& \mathrm{d} \mathfrak{i} \mathcal{L} \mathrm{d} \cong \mathfrak{i} \mathcal{L} Z\left[-2 l\left(w_{0}^{\mathfrak{p}}\right)\right]: \quad \mathcal{D}^{b}\left(\mathcal{O}_{\lambda}\right) \rightarrow \mathcal{D}^{b}\left(\mathcal{O}_{\lambda}\right) \\
& \mathrm{d} \mathcal{L} \mathrm{Z} \mathrm{d} \cong \mathcal{L} \mathrm{Z}\left[-2 l\left(w_{0}^{\mathfrak{p}}\right)\right]: \quad \mathcal{D}^{b}\left(\mathcal{O}_{\lambda}\right) \rightarrow \mathcal{D}^{b}\left(\mathcal{O}_{\lambda}^{\mathfrak{p}}\right)
\end{aligned}
$$

Proof. Using [EW80, Proposition 4.2] we can fix an isomorphism,

$$
\mathrm{d} \mathfrak{i} \mathcal{L} \mathrm{d} \Delta(\lambda) \cong \mathfrak{i} \mathcal{L} Z \Delta(\lambda)\left[-2 l\left(w_{0}^{\mathfrak{p}}\right)\right]
$$

Since Z commutes with projective functors (see e.g. [BFK99, Proposition3]), this isomorphism lifts to an isomorphism on projective modules. We have to verify that it is functorial. Without loss of generality we may assume that $\mathfrak{p}=\mathfrak{p}_{s}$ is the parabolic subalgebra corresponding to a simple reflection $s$. The general case for arbitrary $\mathfrak{p}$ follows then by induction. Associated to $s$, there is a complex of functors

$$
\mathrm{T}_{s} \rightarrow \mathrm{ID} \rightarrow \mathfrak{i Z}
$$

which gives rise to a short exact sequence when applied to projective objects ([KM05, Theorem 4] and [AS03, Proposition 5.4]). Taking the left derived functors we therefore get an isomorphism $\mathcal{L}_{2}(\mathrm{iZ}) \cong \mathcal{L}_{1} \mathrm{~T}_{s}$ (see [KS94, Proposition 1.8.8]). From [AS03, Theorem 4.1] and [MS04, Theorem 1] we have an isomorphism of functors $\mathcal{L}_{1} \mathrm{~T}_{s} \cong \mathrm{diZ}$. The first statement follows, the second is then also clear.

Now we are ready to complete the proof of Proposition 4.4:

Proof of the last part of Proposition 4.4. To prove that $\mathcal{L}\left(C_{w_{0}}\right)^{2}\left[-2 l\left(w_{0}^{\mathfrak{p}}\right)\right]$ is a Serre functor for $\mathcal{D}^{b}\left(\mathcal{O}_{\lambda}^{\mathfrak{p}}\right)$ it is enough to show that for the functor $\mathrm{G}=$ $\mathrm{Z} \mathcal{L}_{2 l\left(w_{0}^{\mathfrak{p}}\right)}\left(C_{w_{0}}\right)^{2} \mathfrak{i}$ we have an isomorphisms, natural in both arguments, as follows:

$$
\operatorname{Hom}_{\mathcal{O}_{\lambda}^{\mathfrak{p}}}\left(P^{\mathfrak{p}}, \mathrm{G} P^{\mathfrak{p}}\right) \cong \operatorname{Hom}_{\mathcal{O}_{\lambda}^{\mathfrak{p}}}\left(P^{\mathfrak{p}}, P^{\mathfrak{p}}\right)^{*}
$$

where $P^{\mathfrak{p}}$ is a projective generator of $\mathcal{O}_{\lambda}^{\mathfrak{p}}$. Without loss of generality we assume $P^{\mathfrak{p}}=\mathrm{ZP}$, where $P$ is a projective generator of $\mathcal{O}_{\lambda}$. We have isomor- 
phisms

$$
\begin{aligned}
\operatorname{Hom}_{\mathcal{O}_{\lambda}^{\mathfrak{p}}}\left(P^{\mathfrak{p}}, \mathrm{G} P^{\mathfrak{p}}\right) & \cong \operatorname{Hom}_{\mathcal{O}_{\lambda}^{\mathfrak{p}}}\left(\mathrm{Z} P, \mathrm{G} P^{\mathfrak{p}}\right) \\
& \cong \operatorname{Hom}_{\mathcal{O}_{\lambda}}\left(\mathfrak{i} \mathrm{Z} P, \mathfrak{i} \mathrm{G}^{\mathfrak{p}}\right) \\
& \cong \operatorname{Hom}_{\mathcal{O}_{\lambda}}\left(P, \mathcal{L}_{2 l\left(w_{0}^{\mathfrak{p}}\right)}\left(C_{w_{0}}\right)^{2} \mathfrak{i} P^{\mathfrak{p}}\right) \\
\text { (by Proposition 4.4 (1)) } & \cong \operatorname{Hom}_{\mathcal{D}^{b}\left(\mathcal{O}_{\lambda}\right)}\left(P, \mathcal{L}\left(C_{w_{0}}\right)^{2} \mathfrak{i} P^{\mathfrak{p}}\left[-2 l\left(w_{0}^{\mathfrak{p}}\right)\right]\right) \\
& \cong \operatorname{Hom}_{\mathcal{D}^{b}\left(\mathcal{O}_{\lambda}\right)}\left(P\left[2 l\left(w_{0}^{\mathfrak{p}}\right)\right], \mathcal{L}\left(C_{w_{0}}\right)^{2} \mathfrak{i} P^{\mathfrak{p}}\right) \\
\text { (by Proposition 4.1) } & \cong \operatorname{Hom}_{\mathcal{D}^{b}\left(\mathcal{O}_{\lambda}\right)}\left(\mathfrak{i} P^{\mathfrak{p}}, P\left[2 l\left(w_{0}^{\mathfrak{p}}\right)\right]\right)^{*} \\
\text { (by adjointness of } \mathfrak{i} \text { and dZd) } & \cong \operatorname{Hom}_{\mathcal{D}^{b}\left(\mathcal{O}_{\lambda}^{\mathfrak{p}}\right)}\left(P^{\mathfrak{p}}, \mathrm{d} \mathcal{L} \mathrm{d} P\left[2 l\left(w_{0}^{\mathfrak{p}}\right)\right]\right)^{*} \\
\text { (by Lemma 4.5) } & \cong \operatorname{Hom}_{\mathcal{D}^{b}\left(\mathcal{O}_{\lambda}^{\mathfrak{p}}\right)}\left(P^{\mathfrak{p}}, \mathcal{L} \mathrm{Z} P\right)^{*} \\
& \cong \operatorname{Hom}_{\mathcal{O}_{\lambda}^{\mathfrak{p}}}\left(P^{\mathfrak{p}}, \mathrm{ZP}\right)^{*} \\
& \cong \operatorname{Hom}_{\mathcal{O}_{\lambda}^{\mathfrak{p}}}\left(P^{\mathfrak{p}}, P^{\mathfrak{p}}\right)^{*},
\end{aligned}
$$

which are natural in both arguments. This completes the proof of Proposition 4.4 .

As an application we get the following nontrivial result

Theorem 4.6. Let $\lambda$ be an integral, regular and dominant weight. We consider the category $\mathcal{O}_{\lambda}^{\mathfrak{p}}$, where $\mathfrak{p} \supset \mathfrak{b}$ is some parabolic subalgebra of $\mathfrak{g}$. Let $Q$ be a basic projective-injective module in $\mathcal{O}_{\lambda}^{\mathfrak{p}}$. Then $\operatorname{End}_{\mathfrak{g}}(Q)$ is symmetric.

For the proof we need the following

Lemma 4.7. In the situation of Theorem 4.6 we have the following: the socle $S$ of $\Delta^{\mathfrak{p}}(\lambda)$ is simple and $\operatorname{Hom}_{\mathfrak{g}}\left(Q, \Delta^{\mathfrak{p}}(\lambda) / S\right)=0$.

Proof. Assume $L$ is a composition factor of $\Delta^{\mathfrak{p}}(\lambda) \operatorname{such}$ that $\operatorname{Hom}_{\mathfrak{g}}(Q, L) \neq 0$. From the latter it follows that the projective cover, $P$, of $L$ is projectiveinjective, hence tilting. Since $L$ is a composition factor of $\Delta^{\mathfrak{p}}(\lambda)$, we have $\operatorname{Hom}_{\mathfrak{g}}\left(\Delta^{\mathfrak{p}}(\lambda), P\right) \neq 0$. Therefore, $L(\lambda)$ appears as a composition factor in $P$. Hence $P=T^{\mathfrak{p}}(\lambda)$ and $L$ is unique. On the other hand

$$
1=\left[T^{\mathfrak{p}}(\lambda): \Delta^{\mathfrak{p}}(\lambda)\right]=\left[P: \Delta^{\mathfrak{p}}(\lambda)\right]=\left[\Delta^{\mathfrak{p}}(\lambda): L\right] .
$$

Since (by [Irv85]) any simple module appearing in the socle of a parabolic Verma module is not annihilated by $\operatorname{Hom}_{\mathfrak{g}}(Q, \bullet)$, the statement follows.

Proof of Theorem 4.6. By Example 2.7 (2) and Proposition 3.5 it is enough to prove that the Serre functor is isomorphic to the identity functor when restricted to the additive subcategory of projective-injective modules. Let $\mathbb{S}=\mathcal{L}\left(C_{w_{0}}\right)^{2}\left[-2 l\left(w_{0}^{\mathfrak{p}}\right)\right]$ be the Serre functor of $\mathcal{D}^{b}\left(\mathcal{O}_{\lambda}^{\mathfrak{p}}\right)$. The idea of the proof is the following: From Proposition 4.4 and Corollary 4.2 we know that $S:=\mathfrak{i} \mathcal{L}_{2 l\left(w_{0}^{\mathfrak{p}}\right)}\left(C_{w_{0}}\right)^{2} \mathrm{Z}: \mathcal{O}_{\lambda} \rightarrow \mathcal{O}_{\lambda}$ is right exact and commutes with translations through walls, even in a natural way as defined in [Kho05]. 
We will construct another right exact functor $\mathrm{G}: \mathcal{O}_{\lambda} \rightarrow \mathcal{O}_{\lambda}$ which again naturally commutes with translations through walls and coincides with $S$ when evaluated at $\Delta(\lambda)$. The main result of [Kho05] states that two right exact additive functors, $F_{1}, F_{2}: \mathcal{O}_{\lambda} \rightarrow \mathcal{O}_{\lambda}$, which agree on $\Delta(\lambda)$, and both naturally commute with translations through walls, are in fact isomorphic. From this fact we will deduce an isomorphism of functors $S \cong \mathrm{G}$. Even more, the main step is to show that we can choose a functor $G$ with the additional property that $\mathrm{G}=\mathfrak{i} \mathrm{G}^{\prime} \mathrm{Z}$ for some $\mathrm{G}^{\prime}: \mathcal{O}_{\lambda}^{\mathfrak{p}} \rightarrow \mathcal{O}_{\lambda}^{\mathfrak{p}}$, and $\mathrm{G}^{\prime}$ is isomorphic to the identity functor when restricted to the category of projective-injective modules in $\mathcal{O}_{\lambda}^{\mathfrak{p}}$. Since $\mathrm{Z}$ is dense and full and $S=\mathfrak{i} \mathcal{L}_{2 l\left(w_{0}^{\mathfrak{p}}\right)}\left(C_{w_{0}}\right)^{2} \mathrm{Z} \cong \mathfrak{i} \mathrm{G}^{\prime} \mathrm{Z}$, we get that $\mathbb{S}$ must be isomorphic to the identity functor on the subcategory of $\mathcal{O}_{\lambda}^{\mathfrak{p}}$ formed by projective-injective modules. This will finally imply the assertion of the theorem.

Let's do the work! We have $\mathbb{S}=\mathcal{L}\left(C_{w_{0}}\right)^{2}\left[-2 l\left(w_{0}^{\mathfrak{p}}\right)\right]$, the Serre functor of $\mathcal{D}^{b}\left(\mathcal{O}_{\lambda}^{\mathfrak{p}}\right)$, given by restriction of $\mathcal{L}\left(C_{w_{0}}\right)^{2}\left[-2 l\left(w_{0}^{\mathfrak{p}}\right)\right]: \mathcal{D}^{b}\left(\mathcal{O}_{\lambda}\right) \rightarrow \mathcal{D}^{b}\left(\mathcal{O}_{\lambda}\right)$. Put $S:=\mathfrak{i} \mathcal{L}_{2 l\left(w_{0}^{\mathfrak{p}}\right)}\left(C_{w_{0}}\right)^{2} \mathrm{Z}$, considered as a functor $\mathcal{O}_{\lambda} \rightarrow \mathcal{O}_{\lambda}$. This functor is clearly right exact and additive. Since $\lambda$ is regular, the category $\mathcal{O}_{\lambda}$ is a category with full projective functors ([Kho05, Proposition 16]) in the sense of [Kho05, Section 2], where the projective functors are given by compositions of translations through walls and their direct summands. Recall from [Kho05, Definition 2] that a functor $G: \mathcal{O}_{\lambda} \rightarrow \mathcal{O}_{\lambda}$ naturally commutes with projective functors if for any projective functor $\theta$, there is an isomorphism of functors $\varphi_{\theta}: \theta G \cong G \theta$ such that the following holds: for any two projective functors $\theta_{1}, \theta_{2}$ and any natural transformation $\alpha \in \operatorname{Hom}\left(\theta_{1}, \theta_{2}\right)$ the following diagram commutes:

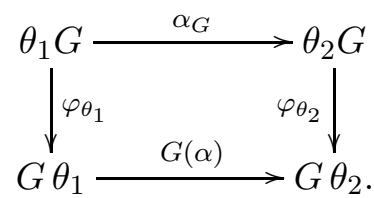

(Note the typos in the original formulation [Kho05, Definition 2].)

Claim 1: The functor $S: \mathcal{O}_{\lambda} \rightarrow \mathcal{O}_{\lambda}$ naturally commutes with projective functors.

Proof of Claim 1. To see this note first that $S \cong \mathcal{L}_{2 l\left(w_{0}^{\mathfrak{p}}\right)}\left(C_{w_{0}}\right)^{2} \mathfrak{i Z}$, where $\mathcal{L}\left(C_{w_{0}}\right)^{2}: \mathcal{D}^{b}\left(\mathcal{O}_{\lambda}\right) \rightarrow \mathcal{D}^{b}\left(\mathcal{O}_{\lambda}\right)$. (This is clear from the definitions.) From [Kho05, Section 6.2] we know that $i \mathrm{Z}$ naturally commutes with projective functors on $\mathcal{O}_{\lambda}$. In [Kho05, Section 6.5] it is proved that twisting functors on $\mathcal{O}_{\lambda}$ naturally commute with projective functors on $\mathcal{O}_{\lambda}$. Corollary 4.2 gives an isomorphism $\left(T_{w_{0}}\right)^{2} \cong\left(C_{w_{0}}\right)^{2}: \mathcal{O}_{\lambda} \rightarrow \mathcal{O}_{\lambda}$, hence $\left(C_{w_{0}}\right)^{2}$ naturally commutes with projective functors. From [Kho05, Lemma 8] it follows that 
$\mathcal{L}_{2 l\left(w_{0}^{\mathfrak{p}}\right)}\left(C_{w_{0}}\right)^{2}$ and $\mathcal{L}_{2 l\left(w_{0}^{\mathfrak{p}}\right)}\left(C_{w_{0}}\right)^{2}$ naturally commute with projective functors, therefore so does $S$, because it is a composition of functors which commute naturally with projective functors. This implies Claim 1.

Let now $J: \mathcal{O}_{\lambda} \rightarrow \mathcal{O}_{\lambda}$ be the partial coapproximation with respect to $M$, where $M=\oplus_{x \in W^{\prime}} P(x \cdot \lambda)$ and

$$
W^{\prime}=\left\{x \in W \mid \mathrm{ZP}(x \cdot \lambda)=0 \text { or } \mathrm{ZP}(x \cdot \lambda) \in \mathcal{O}_{\lambda}^{\mathfrak{p}} \text { is projective-injective }\right\} .
$$

Recall that, when restricted to projective objects, $J$ is nothing else than taking the trace with respect of $M$. The functor $J$ is additive and right exact.

Claim 2: The functor $J: \mathcal{O}_{\lambda} \rightarrow \mathcal{O}_{\lambda}$ naturally commutes with projective functors.

Proof of Claim 2. Let $\theta: \mathcal{O}_{\lambda} \rightarrow \mathcal{O}_{\lambda}$ be a projective functor. We first show that $\theta \operatorname{Tr}_{M} P=\operatorname{Tr}_{M} \theta P$, via the natural inclusions

$$
\theta \operatorname{Tr}_{M} P \hookrightarrow \theta P \hookleftarrow \operatorname{Tr}_{M} \theta P
$$

for any projective module $P \in \mathcal{O}_{\lambda}$. To see this consider the short exact sequence $\operatorname{Tr}_{M} P \hookrightarrow P \rightarrow N$, where $N$ is the canonical quotient, in particular $\operatorname{Hom}_{\mathfrak{g}}(M, N)=0$. We claim that $\operatorname{Hom}_{\mathfrak{g}}(M, \theta N)=0$. Let $\theta^{\prime}$ be the adjoint functor of $\theta$. This is of course again a projective functor and therefore we have the following: If $\mathrm{ZP}(x \cdot \lambda)=0$ then $0=\theta^{\prime} \mathrm{Z} P(x \cdot \lambda) \cong \mathrm{Z} \theta^{\prime} P(x \cdot \lambda)$. If $\mathrm{ZP}(x \cdot \lambda) \neq 0$, but $x \in W^{\prime}$, then $\mathrm{ZP}(x \cdot \lambda)$ is projective-injective in $\mathcal{O}_{\lambda}^{\mathfrak{p}}$, hence so is $\theta^{\prime} \mathrm{Z} P(x \cdot \lambda) \cong \mathrm{Z} \theta^{\prime} P(x \cdot \lambda)$. In particular,

$$
\begin{aligned}
\operatorname{Hom}_{\mathfrak{g}}(M, \theta N) & \cong \operatorname{Hom}_{\mathfrak{g}}\left(\theta^{\prime} M, N\right) \\
& \left.\hookrightarrow \operatorname{Hom}_{\mathfrak{g}}\left(M^{n}, N\right) \quad \text { (for some positive integer } n\right) \\
& =0 .
\end{aligned}
$$

The definition of the trace implies that the projective cover of $\operatorname{Tr}_{M} P$ is a direct summand of some $M^{n}, n \in \mathbb{Z}_{>0}$. From the arguments above it follows that the projective cover of $\theta \operatorname{Tr}_{M} P$ is also a direct summand of some $M^{n}$, $n \in \mathbb{Z}_{>0}$. Altogether, $\theta \operatorname{Tr}_{M} P=\operatorname{Tr}_{M} \theta P$ via the natural inclusions. In other words, we may fix an isomorphism of functors $\varphi_{\theta}: \theta J \cong J \theta$, restricted to the category of projective modules, such that

$$
j_{\theta} \circ \varphi_{\theta}=\theta(j): \theta J \rightarrow \theta,
$$

where $j: J \rightarrow$ ID is the obvious natural transformation. In particular, $J$ commutes with projective functors. We claim that this is already enough to show that $J$ naturally commutes with projective functors. We have to 
check this directly using the original definition [Kho05, Definition 2]: Let $\theta_{1}, \theta_{2}: \mathcal{O}_{\lambda} \rightarrow \mathcal{O}_{\lambda}$ be two projective functors and let $\alpha \in \operatorname{Hom}\left(\theta_{1}, \theta_{2}\right)$ be a natural transformation between them. Consider the following diagram of functors restricted to the additive category formed by all projective objects:

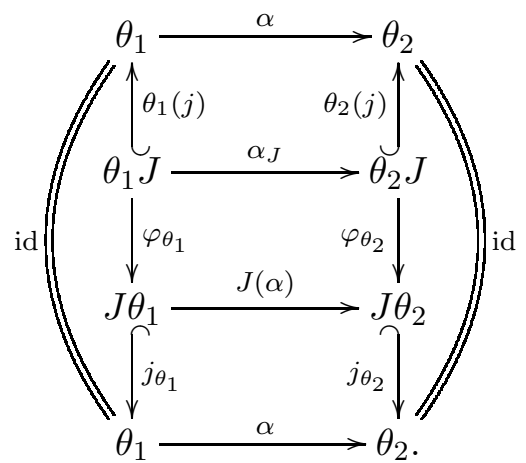

The two "squares", the one on the left hand side and the on the right side, commute because of (4.4). The squares at the top and bottom commute by definition (of a natural transformation). We only have to show that the middle square commutes as well, i.e. $J(\alpha) \circ \varphi_{\theta_{1}}=\varphi_{\theta_{2}} \circ \alpha_{J}$. Since $j_{\theta_{2}}$ is injective (on projective modules) it is enough to show that $j_{\theta_{2}} \circ J(\alpha) \circ \varphi_{\theta_{1}}=$ $j_{\theta_{2}} \circ \varphi_{\theta_{2}} \circ \alpha_{J}$. Since all the other parts of the diagram commute we can calculate

$$
\begin{aligned}
j_{\theta_{2}} \circ J(\alpha) \circ \varphi_{\theta_{1}} & =\alpha \circ j_{\theta_{1}} \circ \varphi_{\theta_{1}} \\
& =\alpha \circ \theta_{1}(j) \\
& =\theta_{2}(j) \circ \alpha_{J} \\
& =j_{\theta_{2}} \circ \varphi_{\theta_{2}} \circ \alpha_{J} .
\end{aligned}
$$

Hence, $J$ commutes naturally with projective functors when restricted to projective objects. Since the involved functors are right exact, Claim 2 follows.

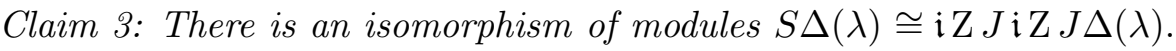

Proof of Claim 3. We first show that $\mathrm{Z} J \Delta(\lambda) \cong \operatorname{soc} \Delta^{\mathfrak{p}}(\lambda)$, the socle of $\Delta^{\mathfrak{p}}(\lambda)$. Define $U$ to be the module which fits into the canonical short exact sequence

$$
0 \rightarrow U \longrightarrow \Delta(\lambda) \longrightarrow \Delta^{p}(\lambda) \rightarrow 0
$$

From Lemma 4.7 we have $\mathrm{Z} J \Delta^{\mathfrak{p}}(\lambda) \cong \operatorname{soc} \Delta^{\mathfrak{p}}(\lambda)$. On the other hand, $Z U=$ 0 by definition, i.e. the projective cover $P_{U}$ of $U$ is annihilated by $\mathrm{Z}$, hence also $\mathrm{Z} J P_{U}=0$. Since $\mathfrak{i Z} J$ is right exact, the sequence (4.5) implies $\mathrm{Z} J U=$ 
0 and $\mathrm{Z} J \Delta(\lambda) \cong \operatorname{soc} \Delta^{\mathfrak{p}}(\lambda)$. From the double centraliser property we have an exact sequence in $\mathcal{O}_{\lambda}^{\mathfrak{p}}$ of the form

$$
0 \rightarrow \Delta(\lambda)^{\mathfrak{p}} \longrightarrow Q_{1} \longrightarrow Q_{2}
$$

where $Q_{1}$ and $Q_{2}$ are projective-injective modules in $\mathcal{O}_{\lambda}^{\mathfrak{p}}$. There is therefore also an exact sequence in $\mathcal{O}_{\lambda}$ of the form

$$
Q_{2} \rightarrow Q_{1} \rightarrow \mathrm{d} \Delta^{\mathfrak{p}}(\lambda) \rightarrow 0 .
$$

Note that $J Q_{1}=Q_{1}, J Q_{2}=Q_{2}$. Hence $\mathfrak{i Z ~} J \mathrm{~d} \Delta^{\mathfrak{p}}(\lambda) \cong \mathrm{d} \Delta^{\mathfrak{p}}(\lambda)$. Altogether we have $\mathfrak{i Z} J \mathfrak{i Z} J \Delta^{\mathfrak{p}}(\lambda) \cong \mathrm{d} \Delta^{\mathfrak{p}}(\lambda)$. The latter is isomorphic to $S \Delta^{\mathfrak{p}}(\lambda)$ by Proposition 4.4. This proves Claim 3.

From [Kho05, Theorem 1] we get the existence of an isomorphism

$$
\alpha: \mathfrak{i Z} J \mathfrak{i Z} \cong S .
$$

By definition, $J$ is isomorphic to the identity functor when restricted to projective-injectives in $\mathcal{O}_{\lambda}^{\mathfrak{p}}$. Therefore, $\alpha$ induces an isomorphism of functors

$$
\mathrm{ID} \cong \mathcal{L}\left(C_{w_{0}}\right)^{2}\left[-2 l\left(w_{0}^{\mathfrak{p}}\right)\right]
$$

when restricted to the category of projective-injective modules in $\mathcal{O}_{\lambda}^{\mathfrak{p}}$. This is exactly the statement that the Serre functor for $\mathcal{D}^{b}\left(\mathcal{O}_{\lambda}^{\mathfrak{p}}\right)$ is isomorphic to the identity when restricted to the additive category formed by projectiveinjective modules. The assertion of the theorem follows then finally from Proposition 3.5.

We get the following consequence

Corollary 4.8. In the situation of Theorem 4.6 the Serre functor for $\mathcal{D}^{b}\left(\mathcal{O}_{\lambda}^{\mathfrak{p}}\right)$ is isomorphic to $\mathcal{L}\left(\left(\operatorname{Coapp}_{Q}\right)^{2}\right)$

Proof. This follows directly from Theorem 4.6 and Theorem 3.7.

\subsection{Harish-Chandra bimodules}

Let ${ }_{\lambda} \mathcal{H}_{\mu}$ be the category of Harish-Chandra bimodules as in Examples 2.7(3), where $\lambda$ and $\mu$ are integral and dominant. Recall the subcategory ${ }_{\lambda} \mathcal{H}_{\mu} \cong$ $A_{\lambda}^{\mu}$-mod. In [BG80] (see also [Jan83, 6.17, 6.23]) it is proved that ${ }_{\lambda} \mathcal{H}_{\mu}^{1}$ is equivalent to the full subcategory $\mathcal{C}_{\lambda}^{\mu}$ of $\mathcal{O}_{\lambda}$ given by all modules $M$, which have an exact presentation,

$$
P_{1} \rightarrow P_{2} \rightarrow M \rightarrow 0,
$$

where $P_{1}$ and $P_{2}$ are projective and the simple modules in their heads are of the form $L(x \cdot \lambda)$, where $x$ is a longest coset representative in $W_{\mu} \backslash W / W_{\lambda}$. Note that this category does not have finite global dimension in general. Nevertheless we have the following 
Proposition 4.9. Let $\lambda, \mu$ be integral dominant weights. Then

(1) $A_{\lambda}^{\mu}$ is Ringel self-dual.

(2) $\mathcal{D}_{\text {perf }}\left(A_{\lambda}^{\mu}\right)$ has a Serre functor, namely $\mathcal{L}\left(C_{w_{0}}\right)^{2}$ (via the identification of $\mathcal{C}_{\lambda}^{\mu}$ with $A_{\lambda}^{\mu}$-mod given by [Aus74, Section 5]).

Proof. From [MS05, Proposition 4.2] and its dual version we get that $C_{w_{0}}$ maps standard modules to costandard modules. From [MS05, Lemma 5.18] it follows that $C_{w_{0}}$ defines an equivalence between the categories of modules with standard flag and modules with costandard flag. In particular, $\operatorname{Ext}_{A_{\lambda}^{\mu}}^{1}\left(C_{w_{0}} P, \nabla\right) \cong \operatorname{Ext}_{A_{\lambda}^{\mu}}^{1}\left(P, C_{w_{0}}^{-1} \nabla\right)=0$ for any costandard module $\nabla$ and any projective $P$. Hence $C_{w_{0}}$ maps a minimal projective generator to a characteristic cotilting module, which is tilting (see e.g. [FKM00, Section 6]). Since it is an equivalence, it preserves the endomorphism ring. The first part of the theorem follows.

To prove the second part of the theorem we use Proposition 4.1. Because of this result and [Gin05, Proposition 20.5.5(i)] it is enough to show that $\mathcal{L}\left(C_{w_{0}}\right)^{2}$ preserves $\mathcal{D}_{\text {perf }}\left(A_{\lambda}^{\mu}\right)$. Since $\mathcal{L}\left(C_{w_{0}}\right)^{2}$ is a Serre functor for $\mathcal{O}$, it sends indecomposable projective modules to the corresponding indecomposable injective modules. However, an injective $A_{\lambda}^{\mu}$-module has a presentation of the form (4.6) by [MS05, Corollary 2.11]. Since all tilting $A_{\lambda}^{\mu}$-modules are also cotilting ([FKM00, Section 6]), it follows that injective $A_{\lambda}^{\mu}$-modules have finite projective dimension. Hence $\mathcal{L}\left(C_{w_{0}}\right)^{2}$ preserves $\mathcal{D}_{\text {perf }}\left(A_{\lambda}^{\mu}\right)$ and the statement follows.

Remark 4.10. From [Dla96, Theorem 2.5] it follows that $A_{\lambda}^{\mu}$ has finite global dimension if and only if the standard and proper standard $A_{\lambda}^{\mu}$-modules coincide (i.e. $A_{\lambda}^{\mu}$ is quasi-hereditary). Using the description of standard modules as in [MS05, Proposition 2.18] it is easy to see that this is the case if and only if $\mu$ is regular or $A_{\lambda}^{\mu}$ is semi-simple.

Remark 4.11. One can also show that for any $N, P \in A_{\lambda}^{\mu}$-mod, where $P$ is projective, the Serre functor $\mathcal{L}\left(C_{w_{0}}\right)^{2}$ from Proposition 4.1 induces a natural isomorphism,

$$
\operatorname{Hom}_{A_{\lambda}^{\mu}}\left(N,\left(C_{w_{0}}\right)^{2} P\right) \cong \operatorname{Hom}_{A_{\lambda}^{\mu}}(P, N)^{*}
$$

\subsection{The category $\mathcal{O}$ for rational Cherednik algebras}

We briefly recall the facts about rational Cherednik algebras which are important in our setup. We refer for example to [GGOR03] for details. Let $V$ be a finite dimensional vector space, $\mathrm{W} \subset \mathrm{GL}(V)$ a finite reflection group, and $\mathbb{C}[\mathrm{W}]$ the group algebra of $\mathrm{W}$ over $\mathbb{C}$. Let $\mathcal{A}$ denote the set of reflection hyperplanes. If $h \in \mathcal{A}$ then $\mathrm{W}_{h}$ denotes the (pointwise) stabiliser of $h$ in $\mathrm{W}$. Let $\gamma: \mathcal{A} \rightarrow \mathbb{C}[\mathrm{W}]$ be a W-equivariant map such that $\gamma(h) \in \mathbb{C}\left[\mathrm{W}_{h}\right] \subset \mathbb{C}[\mathrm{W}]$. 
Associated to the pair $(V, \gamma)$ we have $\mathrm{H}=\mathrm{H}(V, \gamma)$, the corresponding rational Cherednik algebra as defined and studied for example in [GGOR03]. As a vector space, $\mathrm{H}(V, \gamma)$ is isomorphic to $S(V) \otimes \mathbb{C}[\mathrm{W}] \otimes S\left(V^{*}\right)$, where $S(V)$ denotes the algebra of polynomial functions in $V^{*}$ (This is the PBWtheorem [EG02, Theorem 1.3]). The occurring three algebras $S(V), S\left(V^{*}\right)$ and $\mathbb{C}[\mathrm{W}]$ are in fact subalgebras, for the nontrivial commutator relations between them (involving the parameter $\gamma$ ) we refer to [GGOR03], [Gua03]. Let $\mathcal{O}=\mathcal{O}(\mathrm{H}, V, \gamma)$ be the corresponding category $\mathcal{O}$ given by all finitely generated $\mathrm{H}$-modules which are locally $S\left(V^{*}\right)$-finite. This is a highest weight category, where the isomorphism classes of simple modules are in natural bijection with irreducible modules for W. More precisely, if $E$ is an irreducible W-module, then $\Delta(E)=\mathrm{H}(V, \gamma) \otimes_{B} E$, where $B$ is the subalgebra of $\mathrm{H}(V, \gamma)$ generated by $S\left(V^{*}\right)$ and $\mathbb{C}[\mathrm{W}]$. (The action of $p \in S\left(V^{*}\right)$ on $E$ is given by multiplication with $p(0))$. The simple head $L(E)$ of $\Delta(E)$ is the simple module corresponding to $E$.

In general, $\mathrm{H}(V, \gamma)$ is not isomorphic to its opposite algebra $\mathrm{H}(V, \gamma)^{o p p}$, therefore there is no simple preserving duality. However, we have an isomorphism ([GGOR03, Section 4.2]) of algebras $\mathrm{H}(V, \gamma) \cong \mathrm{H}\left(V^{*}, \dagger \circ \gamma\right)^{\text {opp }}$, where $\dagger: \mathrm{W} \rightarrow \mathrm{W}, g \mapsto g^{-1}$ (the isomorphism is given by extending $\dagger$ trivially to $S\left(V^{\star}\right)$ and sending $v \in V$ to $\left.-v\right)$.

With this fixed isomorphism one can define two contravariant functors, namely

- the naive duality ([GGOR03, Proposition 4.7]):

$$
\mathrm{d}_{V, \gamma}: \mathcal{O}(V, \gamma) \rightarrow \mathcal{O}\left(V^{*}, \dagger \circ \gamma\right)
$$

by sending an object $M$ to the largest submodule $\mathrm{d}_{V, \gamma}(M)$ of (the ordinary vector space dual) $M^{*}$ which is locally $S\left(V^{*}\right)$-finite. This is a right $\mathrm{H}(V, \gamma)$-module and becomes a left $\mathrm{H}\left(V^{*}, \dagger \circ \gamma\right)$-module via the fixed isomorphism. This duality sends the simple module $L(E)$ to the simple module $L(\check{E})$ indexed by the dual representation $\check{E}$ of $E$. Projective objects are sent to injectives and standard objects to costandard objects.

- the functor $D_{V, \gamma}$ (see [GGOR03, Proposition 4.10])

$$
D_{V, \gamma}=\operatorname{Ext}_{\mathrm{H}(V, \gamma)}^{\operatorname{dim} V}(\bullet, \mathrm{H}(V, \gamma)): \mathcal{O}(V, \gamma) \rightarrow \mathcal{O}(V, \dagger \circ \gamma)
$$

Conjecture 4.12. Let $\mathrm{H}(V, \gamma)$ be a rational Cherednik algebra with the corresponding category $\mathcal{O}(V, \gamma)$. Then

$$
S=\mathrm{d}_{V^{*}, \gamma^{\dagger}} D_{V^{*}, \gamma} \mathrm{d}_{V, \dagger \circ \gamma} D_{V, \gamma}
$$

is right exact and $\mathcal{L} S$ is a Serre functor. 
To prove this conjecture it would be enough to verify the assumptions in Theorem 3.4, where $F=F_{1} F_{2}, F_{1}=\mathrm{d}_{V, \dagger \circ \gamma} D_{V, \gamma}$ and $F_{2}=\mathrm{d}_{V^{*}, \gamma^{\dagger}} D_{V^{*}, \gamma}$. The fact that $F$ is right exact follows directly from [GGOR03, 4.1]. The assumption (a) follows directly from [GGOR03, Lemma 4.1, Proposition 4.7]. The assumption (b) is proved in [GGOR03, Proposition 5.21]. We do not know if assumption (c) is satisfied. However, a positive answer to the conjecture [GGOR03, Remark 5.20] would imply, via the KnizhnikZamolodchikov-functor, that $F$ is isomorphic to the identity functor on the additive subcategory given by projective-injective objects. Since the corresponding Hecke algebra is symmetric (see e.g. [CIK71, Lemma 5.10]), the conjecture would follow from Proposition 3.5.

Independently of the Conjecture 4.12, we can at least give a description of the corresponding Serre functor in terms of partial coapproximation:

Proposition 4.13. Let $\mathrm{H}(V, \gamma)$ be a rational Cherednik algebra with the corresponding category $\mathcal{O}(V, \gamma)$. Let $Q$ be a basic projective-injective module in $\mathcal{O}(V, \gamma)$. Then the Serre functor of $\mathcal{D}^{b}(\mathcal{O}(V, \gamma))$ is isomorphic to $\mathcal{L}\left(\left(\operatorname{Coapp}_{Q}\right)^{2}\right)$.

Proof. We only have to verify that we are in the situation of Theorem 3.7. We can find a projective-injective module $P_{K Z}$ representing the KnizhnikZamolodchikov-functor (see [GGOR03, Proposition 5.21]). On the other hand the endomorphism ring of $P_{K Z}$ is isomorphic to the Hecke algebra ([GGOR03, Theorem 5.15]), hence symmetric ([CIK71]). It is known that $\mathcal{O}(V, \gamma)$ has the double centraliser property with respect to $P_{K Z}$ ([GGOR03, Theorem 5.16]). Since the naive duality maps a basic projective module to a basic injective module, and $P_{K Z}$ to the corresponding $P_{K Z}$ ([GGOR03, Proposition 5.21]), and $\mathcal{O}(V, \gamma)^{o p p}$ has again a double centraliser property with respect to $P_{K Z}$, we get that $A$ and $A^{o p p}$ have the double centraliser with respect to a basic projective-injective module. From [GGOR03, Proposition 5.21 it follows that such a basic projective-injective module for $A$ is good. Hence, the assumptions of Theorem 3.7 are satisfied. The statement follows.

\section{Projective-injectives in the category $\mathcal{O}^{\mathfrak{p}}\left(\mathfrak{s l}_{n}(\mathbb{C})\right)$}

In the following section we study more carefully projective-injective modules in the parabolic category $\mathcal{O}^{\mathfrak{p}}$, especially, for the Lie algebra $\mathfrak{s l}_{n}=\mathfrak{s l}_{n}(\mathbb{C})$. As already mentioned in the introduction, one of the motivations to consider the category of projective-injective modules in $\mathcal{O}^{\mathfrak{p}}$ is to find a precise connection between Khovanov's categorification of the Jones polynomial ([Kho00]) and the categorification of the Jones polynomial via representation theory 
of the Lie algebra $\mathfrak{s l}_{n}$ (as proposed in [BFK99] and proved in [Str05]). It might be possible to pass directly from one model to the other by connecting the involved algebras directly, because the algebra, used by Khovanov in his categorification, is a quotient of an algebra $A$ such that $A$-mod is equivalent to a certain block of $\mathcal{O}^{\mathfrak{p}}$ for some $\mathfrak{s l}_{n}$ ([Bra02, page 494]). Although, we have a very nice, more or less explicit, description of the algebra $A$ in question ([Bra02, Theorem 1.4.1]), we are interested in more conceptual properties of the algebra. Several conjectures in this direction were formulated by Khovanov in [Kho04]. We want to simplify the problem by using the double centraliser property. In this way, by using the Serre functor, we confirm three conjectures of Khovanov: in Theorem 5.2 we confirm [Kho04, Conjecture 4] concerning the centre of $A$, and in Theorem 5.4 we confirm that the endomorphism algebra of a basic projective-injective module is symmetric, and depends only on the chosen partition of $n$, not on the actually chosen composition of $n$. (The last two conjectures were formulated in a private communication). Furthermore, Theorem 5.4 supports [Kho04, Conjecture 3].

\subsection{On a result of Irving}

Consider the classical triangular decomposition $\mathfrak{s l}_{n}=\mathfrak{n}_{-} \oplus \mathfrak{h} \oplus \mathfrak{n}_{+}$, where $\mathfrak{h}$ is the Cartan subalgebra of all diagonal matrices (with zero trace) and $\mathfrak{n}_{ \pm}$denotes the subalgebra of all upper- and lower-triangular matrices respectively. Given a composition, $\mu=\left(\mu_{1}, \ldots, \mu_{k}\right)$, of $n$ (i.e. $\left.\mu_{1}+\cdots+\mu_{k}=n\right)$, we have the corresponding Young subgroup $S_{\mu}=S_{\mu_{1}} \times S_{\mu_{2}} \times \cdots \times S_{\mu_{k}}$ of $S_{n}$, the latter being the Weyl group of $\mathfrak{s l}_{n}$. Let $\mathcal{O}_{0}^{\mu}$ be the corresponding parabolic subcategory of $\mathcal{O}_{0}\left(\mathfrak{s l}_{n}\right)$ (see Section 4.2). Recall that the simple objects in $\mathcal{O}_{0}$ are in bijection with the elements of $S_{n}$. We denote by $L(w)$ the simple module of highest weight $w(\rho)-\rho$, where $\rho$ denotes the half-sum of the positive roots. For $w \in S_{n}$ we denote by $\Delta(w)$ and $P(w)$ the corresponding Verma and indecomposable projective module in $\mathcal{O}_{0}$. Let $S^{\mu}$ be the set of shortest coset representatives of $S_{\mu} \backslash S_{n}$ and let $w_{\mu}$ be the longest element in $S^{\mu}$. The simple objects in $\mathcal{O}_{0}^{\mu}$ are then the $L(w)$, where $w \in S^{\mu}$. For $w \in S^{\mu}$ we denote by $L^{\mu}(w), \Delta^{\mu}(w)$ and $P^{\mu}(w)$ the corresponding simple, parabolic Verma and indecomposable projective module in $\mathcal{O}_{0}^{\mu}$ respectively. Note that $L^{\mu}(w)=L(w)$ for $w \in S^{\mu}$.

For any $i \in\{1, \ldots, n-1\}$ we denote by $\theta_{i}: \mathcal{O}_{0} \rightarrow \mathcal{O}_{0}$ the translation functor through the $s_{i}$-wall (see e.g. [GJ81, Section 3]). This functor is exact, self-adjoint, and preserves $\mathcal{O}_{0}^{\mu}$. For $w \in S_{n}$ we denote by $\mathcal{L}(w)$ the left cell of the element $w$ (for a definition we refer to [KL79]). Now we can give (for the $\mathfrak{s l}_{n}$ case) an easier proof for the following main result of [Irv85]:

Theorem 5.1. For any composition, $\mu$, of $n$ the following conditions are 
equivalent:

(i) $P^{\mu}(w)$ is injective.

(ii) $w \in \mathcal{L}\left(w_{\mu}\right)$

(iii) $L^{\mu}(w)$ occurs in the socle of some parabolic Verma module $\Delta^{\mu}\left(w^{\prime}\right)$.

Proof. Assume that (i) is satisfied. Since $\mathcal{O}_{0}^{\mu}$ has a simple preserving duality, if $P^{\mu}(w)$ is injective, it is a tilting module in the highest weight category $\mathcal{O}_{0}^{\mu}$ and hence is self-dual. This means that its socle is $L^{\mu}(w)$, which must coincide with the socle of some parabolic Verma module because $P^{\mu}(w)$ has a standard flag. This implies (iii).

Assume that (iii) is satisfied. Since any parabolic Verma module is a submodule of some tilting module, we get that $L^{\mu}(w)$ occurs in the socle of some tilting module. By [CI89], the tilting modules in $\mathcal{O}_{0}^{\mu}$ are exactly direct summands of translations of $L^{\mu}\left(w_{\mu}\right)$. From [Irv85, Proposition 4.3] (this is an easy preparatory result) it follows that $w \in \mathcal{L}\left(w_{\mu}\right)$, that is (ii) is satisfied.

Assume that (ii) is satisfied. Since all $P^{\mu}\left(w^{\prime}\right), w^{\prime} \in \mathcal{L}\left(w_{\mu}\right)$, can be obtained from each other via translations through walls (this follows again from [Irv85, Proposition 4.3]), it is now left to show that there exists $w \in$ $\mathcal{L}\left(w_{\mu}\right)$ such that $P^{\mu}(w)$ is injective. Actually, since we already know that (i) implies (ii), it is enough to show that there exists some projective-injective module in $\mathcal{O}_{0}^{\mu}$. But this one is obtained by translating any simple projective module from the same weight lattice, which exists by [IS88, 3.1] (this is again an easy result).

\subsection{On Khovanov's conjectures}

According to Theorem 5.1, the modules $P^{\mu}(w), w \in \mathcal{L}\left(w_{\mu}\right)$, constitute an exhaustive list of indecomposable projective-injective modules in $\mathcal{O}_{0}^{\mu}$. Let $P_{\mu}=\bigoplus_{w \in \mathcal{L}\left(w_{\mu}\right)} P^{\mu}(w)$ be the basic projective-injective module and set $B_{\mu}=\operatorname{End}_{\mathcal{O}_{0}^{\mu}}\left(P_{\mu}\right)$. As a consequence, we have the following result which confirms [Kho04, Conjecture 4]:

Theorem 5.2. Let $\mu$ be a composition of $n$.

(1) The Loewy lengths of all projective-injective modules in $\mathcal{O}_{0}^{\mu}$ coincide.

(2) $\mathcal{O}_{0}^{\mu}$ satisfies the double centraliser property with respect to a basic projective-injective module, in particular, the restriction induces an isomorphism between the centres of $A_{0}^{\mu}$ and $B_{\mu}$.

Remark 5.3. Theorem 5.2 is true for any semisimple complex Lie algebra

$\mathfrak{g}$ (the proof is exactly the same if one replaces Theorem 5.1 by the main result of [Irv85]). 
Proof. Let $P^{\mu}(w)$ be a projective-injective module. From [Irv85, Proposition 4.3(ii)] it follows that $P^{\mu}(w)$ is a direct summand of some translation of $P^{\mu}\left(w_{\mu}\right)$. Hence to prove the first statement it is enough to show that translations through walls do not increase the Loewy length of projective-injective modules. If $\theta_{i}(L(w)) \neq 0$, then

$$
\operatorname{dim} \operatorname{Hom}_{\mathcal{O}}\left(\theta_{i} P^{\mu}(w), L(w)\right)=\operatorname{dim}_{\operatorname{Hom}}\left(P^{\mu}(w), \theta_{i} L(w)\right)=2
$$

by $\left[\operatorname{Jan} 83,4.12(3), 4.13\left(3^{\prime}\right)\right]$, which implies that $P^{\mu}(w) \oplus P^{\mu}(w)$ is a direct summand of $\theta_{i}\left(P^{\mu}(w)\right)$. Comparing the lengths of the standard filtrations we even get $\theta_{i}\left(P^{\mu}(w)\right) \cong P^{\mu}(w) \oplus P^{\mu}(w)$, in particular, such translations do not produce new projective-injective modules.

Now assume that $\theta_{i}(L(w))=0$. The algebras $A_{0}$ and $A_{0}^{\mu}$, which correspond to $\mathcal{O}_{0}$ and $\mathcal{O}_{0}^{\mu}$ are Koszul ([Soe90], [BGS96], [Bac99]), in particular, they admit a canonical positive grading (the Koszul grading), which we fix. This allows us to consider graded versions of both $\mathcal{O}_{0}$ and $\mathcal{O}_{0}^{\mu}$ (see [BGS96], [Str03a]). In [Str03a] and [BGS96] it was shown that simple modules, Verma modules, parabolic Verma and projective modules in $\mathcal{O}_{0}$ and $\mathcal{O}_{0}^{\mu}$ are gradable. Their graded lifts are unique up to isomorphism and grading shift, therefore we call a lift standard if the head is concentrated in degree zero. In [Str03a] it was shown, that the functors $\theta_{i}$ (as endofunctors of $\mathcal{O}_{0}$ ) are gradable as well. We denote by $\tilde{\theta}_{i}$ the standard graded lift of $\theta_{i}$ (i.e. $\tilde{\theta}_{i}$, applied to a simple module concentrated in degree 0 has socle concentrated in degree 1). Since $\theta_{i}$ preserves $\mathcal{O}_{0}^{\mu}$, the functor $\tilde{\theta}_{i}$ restricts to a graded lift of $\theta_{i}$ on $\mathcal{O}_{0}^{\mu}$.

Let $P^{\text {gr }}$ be the standard graded lift of $P^{\mu}(w)$. Since it has both simple top and simple socle, the radical-, socle- and graded filtrations of $P^{\text {gr }}$ coincide by [BGS96, Proposition 2.4.1]. In particular, $P^{\text {gr }}$ has a unique component of maximal and a unique component of minimal degree. On the other hand, $\tilde{\theta}_{i}(L)$ is concentrated in the degrees $-1,0,1$ for any simple module $L$, concentrated in degree 0 ([Str03a, Theorem 5.1]). This implies that the length of the graded filtration of $\tilde{\theta}_{i}\left(P^{\mathrm{gr}}\right)$ can not exceed the length of the graded filtration of $P^{\mathrm{gr}}$. Hence, the Loewy length of $\theta_{i}\left(P^{\mu}(w)\right)$ does not exceed that of $P^{\mu}(w)$ and the statement (1) follows.

The double centraliser property follows from Corollary 2.6 and the main result of [Irv85] (as formulated in Theorem 5.1). For an algebra, $A$, we denote its centre by $Z(A)$. From the double centraliser property we have

$$
A_{0}^{\mu}=\operatorname{End}_{B_{\mu}}\left(P_{\mu}\right) \text {. }
$$

If $x \in Z\left(A_{0}^{\mu}\right)$, then $x a=a x$ for all $a \in A_{0}^{\mu}$ and hence $x \in B_{\mu}$. On the other hand, every element of $A_{0}^{\mu}$ commutes with each element in $B_{\mu}$ by definition. Hence $x \in Z\left(B_{\mu}\right)$. This implies $Z\left(A_{0}^{\mu}\right) \hookrightarrow Z\left(B_{\mu}\right)$. Because of the left-right symmetry of the double centraliser we finally get $Z\left(A_{0}^{\mu}\right)=Z\left(B_{\mu}\right)$. This completes the proof. 
We formulate now the main result of this section:

Theorem 5.4. (1) Let $\mu$ be a composition of $n$. Then the algebra $B_{\mu}$ is symmetric.

(2) Let $\mu$ and $\nu$ be two compositions of $n$, which give rise to the same partition of $n$. Then $B_{\mu} \cong B_{\nu}$.

Proof. The statement (1) is just a special case of Theorem 4.6.

Let us now prove (2). Without loss of generality we may assume $\mu=$ $\left(\mu_{1}, \ldots, \mu_{k}\right)$ and

$$
\nu=\left(\mu_{1}, \ldots, \mu_{l-1}, \mu_{l+1}, \mu_{l}, \mu_{l+2}, \ldots, \mu_{k}\right)
$$

for some $l \in\{1, \ldots, k-1\}$. Moreover, we assume $\mu_{l}>\mu_{l+1}$.

For any composition, $\tau$, of $n$ Irving and Shelton constructed in [IS88, 3.1] a special weight, $\lambda(\tau)$, with the following property: the simple highest weight module $L(\lambda(\tau)-\rho)$ with the highest weight $\lambda(\tau)-\rho$ is the only simple module in its block of $\mathcal{O}^{\tau}$. From the definition in [IS88, 3.1] it follows immediately that, if $\tau$ and $\tau^{\prime}$ are two compositions of $n$ which give rise to the same partition, then $\lambda(\tau)$ and $\lambda\left(\tau^{\prime}\right)$ are in the same $S_{n}$-orbit, in particular, $L(\lambda(\tau)-\rho)$ and $L\left(\lambda\left(\tau^{\prime}\right)-\rho\right)$ belong to the same block of $\mathcal{O}$.

Now we apply this to the case $\tau=\nu, \tau^{\prime}=\mu$. Let $\mathcal{O}_{\xi}$ be the common block (of $\mathcal{O}$ ) for $L(\lambda(\mu)-\rho)$ and $L(\lambda(\nu)-\rho)$. Although $L(\lambda(\mu)-\rho)$ and $L(\lambda(\nu)-\rho)$ are in the same block of $\mathcal{O}$, we have that the parabolic categories $\mathcal{O}_{\xi}^{\mu}$ and $\mathcal{O}_{\xi}^{\nu}$ are semi-simple containing only one simple object each. Obviously, they are equivalent. However, we would like to construct a functor on $\mathcal{O}$, which gives rise to an equivalence between these categories, and, additionally, commutes with tensoring with finite-dimensional $\mathfrak{s l}_{n}$-modules.

To proceed we will need some general notation. For any transposition $s=s_{i}=(i, i+1)$ in $S_{n}$ we set $\mathcal{O}^{s}=\mathcal{O}^{\beta}$, where $\beta=\left(\beta_{1}, \ldots, \beta_{n-1}\right)$ is the composition of $n$ such that $\beta_{i}=2$ and $\beta_{j}=1$ for all $j \neq i$. Denote by $\mathfrak{i}_{s}: \mathcal{O}^{s} \hookrightarrow \mathcal{O}$ the inclusion functor, and by $\mathrm{Z}_{s}: \mathcal{O} \rightarrow \mathcal{O}^{s}$ the left adjoint to $\mathfrak{i}_{s}$, which is the Zuckerman functor, associated to $s$. Then $\mathrm{dZ}_{s} \mathrm{~d}: \mathcal{O}^{s} \hookrightarrow \mathcal{O}$ is the right adjoint to $\mathfrak{i}_{s}$. It is known that

$$
\mathcal{L}_{i} \mathrm{Z}_{s}=0 \text { for all } i \geq 3, \quad \text { and } \quad \mathcal{L Z}_{s} \cong \mathrm{d} \mathcal{L} \mathrm{Z}_{s} \mathrm{~d}[2],
$$

see [EW80] or [BFK99, Proposition 3] and also Lemma 4.5. Finally, we denote by $\mathfrak{i}_{\mu}: \mathcal{O}^{\mu} \hookrightarrow \mathcal{O}$ and $\mathfrak{i}_{\nu}: \mathcal{O}^{\nu} \hookrightarrow \mathcal{O}$ the inclusion functors and by $\mathrm{Z}_{\mu}: \mathcal{O} \rightarrow \mathcal{O}^{\mu}$ and $\mathrm{Z}_{\nu}: \mathcal{O} \rightarrow \mathcal{O}^{\nu}$ the corresponding left adjoint Zuckerman functors.

Let $m^{\prime}=m_{l}^{\prime}=\mu_{1}+\cdots+\mu_{l-1}$ and $m=m_{l}=m^{\prime}+\mu_{l+1}$, and set $r=\mu_{l}-\mu_{l+1}$. Consider the following element in $S_{n}$ :

$$
w=\left(s_{m^{\prime}+r+1} \ldots s_{m+r}\right) \ldots\left(s_{m^{\prime}+2} \ldots s_{m+1} s_{m^{\prime}+1}\right)\left(s_{m^{\prime}+1} s_{m^{\prime}+2} \ldots s_{m-1} s_{m}\right) .
$$


(for example if $\mu=(2,4)$ then $\nu=(4,2)$ and we have $w=\left(s_{2} s_{3}\right)\left(s_{1} s_{2}\right)$ ). For simplicity we write the above product in the form $w=t_{1} \ldots t_{p}$ (thus $p=r \mu_{l+1}, t_{1}=s_{m^{\prime}+r+1}$ and so on). For $i=1, \ldots, p$ we set $w_{i}=t_{i} \ldots t_{p}$, and $w_{p+1}=e$. The element $w$ is constructed such that $w(\lambda(\nu))=\lambda(\mu)$ and $w_{i}(\lambda(\nu))>w_{i+1}(\lambda(\nu))$ for all $i=1, \ldots, p$. Define

$$
\begin{array}{ll}
F=\mathcal{L} \mathrm{Z}_{\mu} \mathfrak{i}_{t_{1}} \mathcal{L} \mathrm{Z}_{t_{1}} \mathfrak{i}_{t_{2}} \mathcal{L} \mathrm{Z}_{t_{2}} \ldots \mathfrak{i}_{t_{p}} \mathcal{L} \mathrm{Z}_{t_{p}} \mathfrak{i}_{\nu}[-l(w)] & : \mathcal{D}^{b}\left(\mathcal{O}^{\nu}\right) \rightarrow \mathcal{D}^{b}\left(\mathcal{O}^{\mu}\right) \\
G=\mathcal{L} \mathrm{Z}_{\nu} \mathfrak{i}_{t_{p}} \mathcal{L} \mathrm{Z}_{t_{p}} \mathfrak{i}_{t_{p-1}} \mathcal{L} \mathrm{Z}_{t_{p-1}} \ldots \mathfrak{i}_{t_{1}} \mathcal{L} \mathrm{Z}_{t_{1}} \mathfrak{i}_{\mu}[-l(w)] & : \mathcal{D}^{b}\left(\mathcal{O}^{\mu}\right) \rightarrow \mathcal{D}^{b}\left(\mathcal{O}^{\nu}\right)
\end{array}
$$

Both, $F$ and $G$, commute with tensoring with finite-dimensional $\mathfrak{s l}_{n}$-modules ([EW80, Proposition 2.2 and Proposition 3.7], see also [BFK99, Proposition 3]). Further, $F$ is both left and right adjoint to $G$ by (5.1).

Claim: The functors $F$ and $G$ define, via restriction, mutually inverse equivalences $F: \mathcal{O}_{\xi}^{\nu} \rightarrow \mathcal{O}_{\xi}^{\mu}$ and $G: \mathcal{O}_{\xi}^{\mu} \rightarrow \mathcal{O}_{\xi}^{\nu}$.

Proof of the Claim. Since $F$ and $G$ are adjoint to each other and both $\mathcal{O}_{\xi}^{\mu}$ and $\mathcal{O}_{\xi}^{\nu}$ are semi-simple, it is enough to show that

$$
F(L(\lambda(\nu)-\rho))=L(\lambda(\mu)-\rho) .
$$

To prove this we first note that for a simple reflection, $s$, and for a dominant integral weight, $\lambda$, we have

$$
\mathcal{L Z}_{s}(L(x \cdot \lambda))= \begin{cases}L(x \cdot \lambda) \oplus L(x \cdot \lambda)[2], & s x \cdot \lambda<x \cdot \lambda, \\ \bigoplus_{y: y>s x} L(y \cdot \lambda)^{a_{y}}[1], & s x \cdot \lambda=x \cdot \lambda, \\ L(s x \cdot \lambda)[1] \oplus \bigoplus_{y: y>s x} L(y \cdot \lambda)^{a_{y}[1],} & \text { otherwise, }\end{cases}
$$

where $a_{y} \in\{0,1, \ldots\}$. To see this, let $\mathrm{T}_{s}: \mathcal{O} \rightarrow \mathcal{O}$ be the twisting functor, associated with $s$ (as in Subsection 4.1). In [KM05, Theorem 4] (see also [MS04, Proposition 2.3]) it is shown that there exists a natural transformation, $\operatorname{can}_{s}: \mathrm{T}_{s} \rightarrow \mathrm{ID}$, non-vanishing on Verma modules. In [MS04, Theorem 1(3)] it is proved that the kernel of $\operatorname{can}_{s}$ is isomorphic to $\mathcal{L}_{1} Z_{s}$. Now (5.2) follows from the Kazhdan-Lusztig conjectures, see [AS03, Theorem 6.3 and Theorem 7.8] for details. (Note that the assumption for $L^{\prime}$ to be $s$-finite is missing in the formulation of [AS03, Theorem 6.3(3)].)

For $i=1, \ldots, p$ set

$$
F_{i}=\mathcal{L} \mathrm{Z}_{t_{i}} \mathfrak{i}_{t_{2}} \mathcal{L} \mathrm{Z}_{t_{2}} \ldots \mathfrak{i}_{t_{p}} \mathcal{L} \mathrm{Z}_{t_{p}} \mathfrak{i}_{\nu}[-(p-i+1)]: \mathcal{D}^{b}\left(\mathcal{O}^{\nu}\right) \rightarrow \mathcal{D}^{b}\left(\mathcal{O}^{t_{i}}\right) .
$$

From (5.2) it follows by induction that

$$
F_{i} L(\lambda(\tau)-\rho)=L\left(w_{i}(\lambda(\tau))-\rho\right) \oplus \bigoplus_{y: y>w_{i}} L(y \cdot \lambda(\tau))^{a_{y}^{(i)}}\left[b_{y}^{(i)}\right]
$$


where $a_{y}^{(i)} \in\{0,1, \ldots\}, b_{y}^{(i)} \in\{1, \ldots\}$ for all $i$. In particular, we have

$$
\mathcal{L} Z_{\mu} \mathfrak{i}_{t_{1}} F_{1} L(\lambda(\tau)-\rho)=L(\lambda(\mu)-\rho)
$$

since $\mathcal{O}_{\xi}^{\mu}$ is semi-simple and the claim follows.

Let us now sum up what we know. We have an adjoint pair, $(F, G)$, of functors between $\mathcal{D}^{b}\left(\mathcal{O}^{\nu}\right)$ and $\mathcal{D}^{b}\left(\mathcal{O}^{\mu}\right)$, which commute with tensoring with finite-dimensional $\mathfrak{s l}_{n}$-modules and induce mutually inverse equivalences, when restricted to $\mathcal{O}_{\xi}^{\nu}$ and $\mathcal{O}_{\xi}^{\mu}$. On the other hand, there is a finite dimensional $\mathfrak{s l}_{n}$-module, $E$, such that $E \otimes L(\lambda(\nu)-\rho)$ contains $P_{\nu}$ as a direct summand, and $E \otimes L(\lambda(\mu)-\rho)$ contains $P_{\mu}$ as a direct summand (this follows from Theorem 5.2, for the explicit statement see [Irv85, Proposition 4.3(ii)]). Therefore the adjunction morphisms $F G \rightarrow$ ID and ID $\rightarrow G F$ are isomorphisms when evaluated at $P_{\mu}$ and $P_{\nu}$ respectively. Hence $F$ and $G$ define mutually inverse equivalences between the corresponding additive categories of projective-injective modules. This completes the proof of Theorem 5.4 .

We have the following direct consequence, a part of which was also obtained in [Kho04, Section 6] by establishing a derived equivalence between $\mathcal{O}_{\xi}^{\mu}$ and $\mathcal{O}_{\xi}^{\nu}$ using a geometric argument:

Corollary 5.5. The centres of $B_{\mu}, B_{\nu}, \mathcal{O}_{\xi}^{\mu}$ and $\mathcal{O}_{\xi}^{\nu}$ are all isomorphic.

Remark 5.6. Since the Kazhdan-Lusztig left cell modules for the IwahoriHecke algebra $\mathcal{H}_{n}$ of the symmetric group are exactly the irreducible modules, Theorem 5.1 can be used to "categorify" these irreducible modules: Let $\lambda$ be a partition of $n$. Consider the abelian category of modules, admitting a 2-step presentation by projective-injective modules in the parabolic category $\mathcal{O}$ for $\mathfrak{s l}_{n}$, associated with $\lambda$. This abelian category is invariant under the action of translations through walls. The action of these translation functors gives rise to a categorification of the Specht module $S_{\lambda}$ for the symmetric group $S_{n}$. The graded version of the above result (in the sense of [Str03a]) gives rise to a categorification of the Specht module $S_{\lambda}$ for $\mathcal{H}_{n}$. The details will appear in [KMS].

\section{References}

[ÁHLU00] I. Ágoston, D. Happel, E. Lukács, and L. Unger, Finitistic dimension of standardly stratified algebras, Comm. Algebra 28 (2000), no. 6, 2745-2752.

[AL03] H. H. Andersen and N. Lauritzen, Twisted Verma modules, Studies in memory of Issai Schur, Progr. Math., vol. 210, Birkhäuser, 2003, pp. 1-26. 
[AR91] M. Auslander and I. Reiten, Applications of contravariantly finite subcategories, Adv. Math. 86 (1991), no. 1, 111-152.

[Ark97] S. M. Arkhipov, Semi-infinite cohomology of associative algebras and bar duality, Internat. Math. Res. Notices (1997), no. 17, 833-863.

[AS03] H. H. Andersen and C. Stroppel, Twisting functors on $\mathcal{O}$, Represent. Theory 7 (2003), 681-699 (electronic).

[Aus74] M. Auslander, Representation theory of Artin algebras. I, II, Comm. Algebra 1 (1974), 177-268.

[Bac99] E. Backelin, Koszul duality for parabolic and singular category $\mathcal{O}$, Represent. Theory 3 (1999), 139-152 (electronic).

[BBM04] A. Beilinson, R. Bezrukavnikov, and I. Mirković, Tilting exercises, Mosc. Math. J. 4 (2004), no. 3, 547-557, 782.

[BFK99] J. Bernstein, I. Frenkel, and M. Khovanov, A categorification of the Temperley-Lieb algebra and Schur quotients of $U\left(\mathfrak{s l}_{2}\right)$ via projective and Zuckerman functors, Selecta Math. (N.S.) 5 (1999), no. 2, 199-241.

[BG80] J. N. Bernstein and S. I. Gel'fand, Tensor products of finite- and infinite-dimensional representations of semisimple Lie algebras, Compositio Math. 41 (1980), no. 2, 245-285.

[BGG76] I. N. Bernstein, I. M. Gel'fand, and S. I. Gel'fand, A certain category of $\mathfrak{g}$-modules, Funkcional. Anal. i Priložen. 10 (1976), no. $2,1-8$.

[BGS96] A. Beilinson, V. Ginzburg, and W. Soergel, Koszul duality patterns in representation theory, J. Amer. Math. Soc. 9 (1996), no. $2,473-527$.

[Bir74] J. S. Birman, Braids, links, and mapping class groups, Princeton University Press, 1974, Annals of Mathematics Studies, No. 82.

[BK89] A. I. Bondal and M. M. Kapranov, Representable functors, Serre functors, and reconstructions, Izv. Akad. Nauk SSSR Ser. Mat. 53 (1989), no. 6, 1183-1205, 1337.

[Bra02] T. Braden, Perverse sheaves on Grassmannians, Canad. J. Math. 54 (2002), no. 3, 493-532. 
[CI89] D. H. Collingwood and R. S. Irving, A decomposition theorem for certain self-dual modules in the category $\mathcal{O}$, Duke Math. J. 58 (1989), no. 1, 89-102.

[CIK71] C. W. Curtis, N. Iwahori, and R. Kilmoyer, Hecke algebras and characters of parabolic type of finite groups with $(B, N)$-pairs, Inst. Hautes Études Sci. Publ. Math. (1971), no. 40, 81-116.

[CPS88] E. Cline, B. Parshall, and L. Scott, Finite-dimensional algebras and highest weight categories, J. Reine Angew. Math. 391 (1988), 85-99.

[CPS96] - Stratifying endomorphism algebras, Mem. Amer. Math. Soc. 124 (1996), no. 591.

[Dla96] V. Dlab, Quasi-hereditary algebras revisited, An. Ştiinţ. Univ. Ovidius Constanţa Ser. Mat. 4 (1996), no. 2, 43-54, Representation theory of groups, algebras, and orders (Constanţa, 1995).

[Dla00] - Properly stratified algebras, C. R. Acad. Sci. Paris Sér. I Math. 331 (2000), no. 3, 191-196.

[DR89] V. Dlab and C. M. Ringel, Quasi-hereditary algebras, Illinois J. Math. 33 (1989), no. 2, 280-291.

[DX94] B. Deng and C. Xi, Quasi-hereditary algebras which are dual extensions of algebras, Comm. Algebra 22 (1994), no. 12, 47174735 .

[EG02] P. Etingof and V. Ginzburg, Symplectic reflection algebras, Calogero-Moser space, and deformed Harish-Chandra homomorphism, Invent. Math. 147 (2002), no. 2, 243-348.

[EW80] T. J. Enright and N. R. Wallach, Notes on homological algebra and representations of Lie algebras, Duke Math. J. 47 (1980), no. $1,1-15$.

[FKM00] V. Futorny, S. König, and V. Mazorchuk, $\mathcal{S}$-subcategories in $\mathcal{O}$, Manuscripta Math. 102 (2000), no. 4, 487-503.

[Fri04] A. Frisk, Dlab's theorem and tilting modules for stratified algebras, Tech. report, University of Uppsala, 2004.

[Gab62] P. Gabriel, Des catégories abéliennes, Bull. Soc. Math. France 90 (1962), 323-448. 
[GGOR03] V. Ginzburg, N. Guay, E. Opdam, and R. Rouquier, On the category $\mathcal{O}$ for rational Cherednik algebras, Invent. Math. 154 (2003), no. 3, 617-651.

[Gin05] V. Ginzburg, Lectures on noncommutative geometry, arXiv:math.AG/0506603, 2005.

[GJ81] O. Gabber and A. Joseph, Towards the Kazhdan-Lusztig conjecture, Ann. Sci. École Norm. Sup. (4) 14 (1981), no. 3, 261-302.

[Gua03] N. Guay, Projective modules in the category $\mathcal{O}$ for the Cherednik algebra, J. Pure Appl. Algebra 182 (2003), no. 2-3, 209-221.

[Hap88] D. Happel, Triangulated categories in the representation theory of finite-dimensional algebras, London Mathematical Society Lecture Note Series, vol. 119, Cambridge University Press, 1988.

[Hap91] — Auslander-Reiten triangles in derived categories of finite-dimensional algebras, Proc. Amer. Math. Soc. 112 (1991), no. 3, 641-648.

[Irv85] R. Irving, Projective modules in the category $\mathcal{O}_{S}$ : self-duality, Trans. Amer. Math. Soc. 291 (1985), no. 2, 701-732.

[Irv93] _ Shuffled Verma modules and principal series modules over complex semisimple Lie algebras, J. London Math. Soc. (2) 48 (1993), no. 2, 263-277.

[IS88] R. S. Irving and B. Shelton, Loewy series and simple projective modules in the category $\mathcal{O}_{S}$, Pacific J. Math. 132 (1988), no. 2, 319-342.

[Jan83] J. C. Jantzen, Einhüllende Algebren halbeinfacher Lie-Algebren, Ergebnisse der Mathematik und ihrer Grenzgebiete (3), vol. 3, Springer-Verlag, Berlin, 1983.

[Jos82] A. Joseph, The Enright functor on the Bernstein-Gel'fandGel' fand category $\mathcal{O}$, Invent. Math. 67 (1982), no. 3, 423-445.

[Kho00] M. Khovanov, A categorification of the Jones polynomial, Duke Math. J. 101 (2000), no. 3, 359-426.

[Kho04] Crossingless matchings and the cohomology of $(n, n)$ Springer varieties, Commun. Contemp. Math. 6 (2004), no. 4, $561-577$.

[Kho05] O. Khomenko, Categories with projective functors, Proc. London Math. Soc. (3) 90 (2005), no. 3, 711-737. 
[KK99] M. Klucznik and S. König, Characteristic tilting modules over Quasi-hereditary algebras, SFB343 Universität Bielefeld, 1999.

[KL79] D. Kazhdan and G. Lusztig, Representations of Coxeter groups and Hecke algebras, Invent. Math. 53 (1979), no. 2, 165-184.

[KM02] S. König and V. Mazorchuk, Enright's completions and injectively copresented modules, Trans. Amer. Math. Soc. 354 (2002), no. 7, 2725-2743 (electronic).

[KM05] O. Khomenko and V. Mazorchuk, On Arkhipov's and Enright's functors, Math. Z. 249 (2005), no. 2, 357-386.

[KMS] M. Khovanov, V. Mazorchuk, and C. Stroppel, Categorification of irreducible representations of symmetric groups and their Hecke algebras, in preparation.

[KS94] M. Kashiwara and P. Schapira, Sheaves on manifolds, Grundlehren der Mathematischen Wissenschaften, vol. 292, SpringerVerlag, 1994.

[KSX01] S. König, I. H. Slungard, and C. Xi, Double centralizer properties, dominant dimension, and tilting modules, J. Algebra 240 (2001), no. 1, 393-412.

[Lep77] J. Lepowsky, A generalization of the Bernstein-Gelfand-Gelfand resolution, J. Algebra 49 (1977), no. 2, 496-511.

[MS04] V. Mazorchuk and C. Stroppel, On functors associated to a simple root, arXiv:math.RT/0410339, 2004.

[MS05]__ Translation and shuffling of projectively presentable modules and a categorification of a parabolic Hecke module, Trans. Amer. Math. Soc. 357 (2005), no. 7, 2939-2973.

[RC80] A. Rocha-Caridi, Splitting criteria for $\mathfrak{g}$-modules induced from

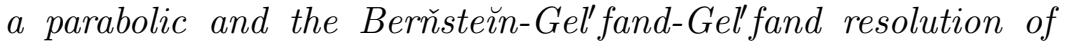
a finite-dimensional, irreducible $\mathfrak{g}$-module, Trans. Amer. Math. Soc. 262 (1980), no. 2, 335-366.

[Rin91] C. M. Ringel, The category of modules with good filtrations over a quasi-hereditary algebra has almost split sequences, Math. Z. 208 (1991), no. 2, 209-223.

[RVdB02] I. Reiten and M. Van den Bergh, Noetherian hereditary abelian categories satisfying Serre duality, J. Amer. Math. Soc. 15 (2002), no. 2, 295-366 (electronic). 
[Soe90] W. Soergel, Kategorie $\mathcal{O}$, perverse Garben und Moduln über den Koinvarianten zur Weylgruppe, J. Amer. Math. Soc. 3 (1990), no. 2, 421-445.

[Soe97] — Charakterformeln für Kipp-Moduln über Kac-MoodyAlgebren, Represent. Theory 1 (1997), 115-132 (electronic).

[Str03a] C. Stroppel, Category $\mathcal{O}:$ Gradings and Translation functors, Journal of Algebra 268 (2003), no. 1, 301-326.

[Str03b] Category $\mathcal{O}$ : quivers and endomorphism rings of projectives, Represent. Theory 7 (2003), 322-345 (electronic).

[Str05] _ Categorification of the Temperley-Lieb category, tangles, and cobordisms via projective functors, Duke Math. J. 126 (2005), no. 3, 547-596.

Volodymyr Mazorchuk, Department of Mathematics, Uppsala University, Box 480, 751 06, Uppsala, SWEDEN, e-mail: mazor@math.uu.se, web: "http://www.math.uu.se/mazor/".

Catharina Stroppel, Department of Mathematics, University of Glasgow, University Gardens, Glasgow G12 8QW, UK,

e-mail: cs@maths.gla.ac.uk 